\title{
Some properties of implicit impulsive coupled system via $\varphi$-Hilfer fractional operator
}

\author{
Mohammed A. Almalahi ${ }^{1,2^{*}}$ (D) and Satish K. Panchal ${ }^{1}$
}

\section{"Correspondence:}

aboosama736242107@gmail.com

'Department of Mathematics, Dr.

Babasaheb Ambedkar Marathwada

University, Aurangabad, (M.S), 431001, India

${ }^{2}$ Department of Mathematics,

Hajjah University, Hajjah, Yemen

\section{Springer}

\begin{abstract}
The major goal of this work is investigating sufficient conditions for the existence and uniqueness of solutions for implicit impulsive coupled system of $\varphi$-Hilfer fractional differential equations (FDEs) with instantaneous impulses and terminal conditions. First, we derive equivalent fractional integral equations of the proposed system. Next, by employing some standard fixed point theorems such as Leray-Schauder alternative and Banach, we obtain the existence and uniqueness of solutions. Further, by mathematical analysis technique we investigate the Ulam-Hyers (UH) and generalized UH (GUH) stability of solutions. Finally, we provide a pertinent example to corroborate the results obtained.
\end{abstract}

MSC: 34A08; 34B15; 34A12; 47H10

Keywords: $\varphi$-Hilfer FDEs; Terminal conditions; Impulsive coupled system; Existence theory; Fixed point theorem

\section{Introduction}

Fractional differential equations (FDEs) have attracted the interest of researchers from various disciplines as they are a useful tool in modeling the dynamics of numerous physical systems and have applications in many fields of applied sciences, engineering and technical sciences, and so on. For further details, see [26, 36, 38, 40]. There are various definitions of fractional calculus (FC) used in FDEs for modeling and describing the memory accurately. Among the famous operators of this calculus, there are Riemann-Liouville, Riemann, Grünwald-Letnikov, Caputo, Hilfer, and Hadamard, which are the most used. For more detail, we refer the readers to $[1-3,21,22,24,25,33,34,36,41]$. There is a prominent and noticeable interest in the investigation of qualitative characteristics of solutions (existence, uniqueness, stability) of FDEs. For applications and recent work, we refer the readers to $[4,7,14,18,37,42,43]$.

In recent years, the impulsive fractional differential equations have become an important and successful tool in modeling some physical phenomena that have sudden changes and have discontinuous jumps by imposing impulsive conditions on the fractional differ-

(c) The Author(s) 2021. This article is licensed under a Creative Commons Attribution 4.0 International License, which permits use, sharing, adaptation, distribution and reproduction in any medium or format, as long as you give appropriate credit to the original author(s) and the source, provide a link to the Creative Commons licence, and indicate if changes were made. The images or other third party material in this article are included in the article's Creative Commons licence, unless indicated otherwise in a credit line to the material. If material is not included in the article's Creative Commons licence and your intended use is not permitted by statutory regulation or exceeds the permitted use, you will need to obtain permission directly from the copyright holder. To view a copy of this licence, visit http://creativecommons.org/licenses/by/4.0/. 
ential equations at discontinuity points. For applications and recent work, we refer the readers to $[8,9,12,13,17,27,28,32,44]$.

On the other side, the study of coupled systems involving FDEs is also important as such systems occur in various problems of applied nature. For some theoretical works on coupled systems of FDEs, we refer to series of papers [11, 16, 19, 20, 23, 30].

The topic of system stability is one of the most important qualitative characteristics of a solution, but to our knowledge, the results on UH and UHR stability of solutions for implicit impulsive coupled systems are very few in the literature.

Very recently, Kharade and Kucche [35] studied the existence and uniqueness of solutions and UHML stability for the following impulsive implicit problem:

$$
\begin{cases}\mathcal{D}_{a^{+}}^{\mathfrak{y}, \mathfrak{p} ; \varphi} u(\sigma)=f\left(\sigma, u(\sigma), u(h(\sigma)), \mathcal{D}_{a^{+}}^{\mathfrak{y}, \mathfrak{p} ; \varphi} y(\sigma)\right), & \sigma \in \mathcal{J}:=[0, T], \sigma \neq \sigma_{k}, k=1, \ldots, p, \\ \left.\Delta I_{0^{+}}^{1-\gamma, \varphi} u\left(\sigma_{k}\right)\right|_{\sigma=\sigma_{k}}=J_{k} u\left(\sigma_{k}^{-}\right), & k=1, \ldots, p, \\ I_{0^{+}}^{1-\gamma, \varphi} u(0)=u_{0}, & \sigma \in[-r, 0], \\ u(\sigma)=\phi(\sigma), & \end{cases}
$$

where $\mathcal{D}_{a^{+}}^{\mathfrak{y}, \mathfrak{p} ; \varphi}$ denotes the $\varphi$-Hilfer fractional derivative (FD) of order $\mathfrak{y} \in(0,1)$ and type $\mathfrak{p} \in[0,1]$, and $f: \mathcal{J} \times \mathbb{R} \times \mathbb{R} \times \mathbb{R} \longrightarrow \mathbb{R}$ is a continuous function. Via standard fixed point theorems, Ahmed et al. [10] studied the existence, uniqueness, and different kinds of stability of the following switched coupled implicit $\varphi$-Hilfer fractional differential system:

$$
\begin{cases}\mathcal{D}_{a^{+}}^{\mathfrak{y}, \mathfrak{p}, \varphi} \mathfrak{u}(\sigma)=f\left(\sigma, \mathfrak{u}(\sigma), \mathcal{D}_{a^{+}}^{\mathfrak{y}, \mathfrak{p}, \varphi} \vartheta(\sigma)\right), & \sigma \in J:=[0, T], \\ \mathcal{D}_{a^{+}, \mathfrak{p}, \varphi} \vartheta(\sigma)=g\left(\sigma, \mathcal{D}_{a^{+}}^{\mathfrak{y}, \mathfrak{p} \varphi} \mathfrak{u}(\sigma), \vartheta(\sigma)\right), & \sigma \in J:=[0, T], \\ I_{a^{+}}^{1-\gamma, \varphi} \mathfrak{u}(a)=\mathfrak{u}_{a}, \quad I_{a^{+}}^{1-\gamma, \varphi} \vartheta(a)=\vartheta_{a}, \mathfrak{u}_{a}, & \vartheta_{a} \in \mathbb{R},\end{cases}
$$

where $\mathcal{D}_{a^{+}}^{\mathfrak{y}, \mathfrak{p}, \varphi}$ denotes the $\varphi$-Hilfer FD of order $\mathfrak{y} \in(0,1)$ and type $\mathfrak{p} \in[0,1]$, and $f, g:[0, T] \times$ $\mathbb{R} \times \mathbb{R} \longrightarrow \mathbb{R}$ are continuous functions.

Abdo et al. [5], via standard fixed point theorems, studied the existence and uniqueness of the following impulsive problem:

$$
\begin{cases}{ }^{A B} \mathcal{D}_{[\sigma]}^{\mathfrak{y}} u(\sigma)=f(\sigma, u(\sigma)), & \sigma \in \mathcal{J}:=[0, T], \sigma \neq \sigma_{k}, k=1, \ldots, m, \\ \left.\Delta u\right|_{\sigma=\sigma_{k}}=I_{k} u\left(\sigma_{k}^{-}\right), & k=1, \ldots, m, \\ u(0)=u_{0} . & \end{cases}
$$

On the other hand, Almalahi et al. [15] studied the existence and uniqueness of solution for the following FDEs:

$$
\left\{\begin{array}{l}
\mathcal{D}_{a^{+}}^{\mathfrak{y}, \mathfrak{p} ; \varphi} y(\sigma)=f\left(\sigma, y(\sigma), \mathcal{D}_{a^{+}}^{\mathfrak{y}, \mathfrak{p} ; \varphi} y(\sigma)\right), \quad \sigma \in(a, T], a>0, \\
y(T)=w \in \mathbb{R}
\end{array}\right.
$$

where $\mathcal{D}_{a^{+}}^{\mathfrak{y}, \mathfrak{p} ; \varphi}$ is the $\varphi$-Hilfer FD of order $\mathfrak{y} \in(0,1)$ and type $\mathfrak{p} \in[0,1]$. 
Abdo et al. [6] studied the existence, uniqueness, and UH stability of the following system:

$$
\left\{\begin{array}{l}
\mathcal{D}_{a^{+}}^{\mathfrak{y}_{1}, \mathfrak{p}_{1} ; \varphi} y(\sigma)=f_{1}(\sigma, y(\sigma)), \quad \sigma \in(a, T], a>0 \\
\mathcal{D}_{a^{+}}^{\mathfrak{y}_{2}, \mathfrak{p}_{2} ; \varphi} x(\sigma)=f_{2}(\sigma, y(\sigma)) \\
y(T)=w_{1} \in \mathbb{R} \\
x(T)=w_{2} \in \mathbb{R}
\end{array}\right.
$$

where $\mathcal{D}_{a^{+}}^{\mathfrak{y}_{1}, \mathfrak{p} ; \varphi}, \mathcal{D}_{a^{+}}^{\mathfrak{y}_{2}, \mathfrak{p} ; \varphi}$ are the $\varphi$-Hilfer FDs of orders $\mathfrak{y}_{1}, \mathfrak{y}_{2} \in(0,1)$ and type $\mathfrak{p} \in[0,1]$.

Motivated by the preceding works, in this paper, we investigate the existence, uniqueness, and UH stability for more general implicit impulsive coupled systems of $\varphi$-Hilfer FDEs:

$$
\left\{\begin{array}{l}
\mathcal{D}_{[\sigma]}^{\mathfrak{y}, \mathfrak{p}, \varphi} \mathfrak{u}(\sigma)=f\left(\sigma, \mathfrak{u}(\sigma), \mathcal{D}_{[\sigma]}^{\mathfrak{y}, \mathfrak{p}, \varphi} \vartheta(\sigma)\right), \quad \sigma \in J:=[0, T], \sigma \neq \sigma_{k}, k=1, \ldots, m, \\
\mathcal{D}_{[\sigma]}^{\mathfrak{y}, \mathfrak{p}, \varphi} \vartheta(\sigma)=g\left(\sigma, \mathcal{D}_{[\sigma]}^{\mathfrak{y}, \mathfrak{p}, \varphi} \mathfrak{u}(\sigma), \vartheta(\sigma)\right), \quad \sigma \in J:=[0, T], \sigma \neq \sigma_{k}, k=1, \ldots, m, \\
\left.\Delta \mathfrak{u}\right|_{\sigma=\sigma_{k}}=Z_{k} \mathfrak{u}\left(\sigma_{k}^{-}\right), \quad k=1, \ldots, m,\left.\Delta \vartheta\right|_{\sigma=\sigma_{k}}=Z_{k} \vartheta\left(\sigma_{k}^{-}\right), k=1, \ldots, m, \\
\mathfrak{u}(T)=w_{1}, \quad \vartheta(T)=w_{2},
\end{array}\right.
$$

where $\mathcal{D}_{[\sigma]}^{\mathfrak{y}, \mathfrak{p}, \varphi}$ denotes the $\varphi$-Hilfer FD of order $\mathfrak{y} \in(0,1)$ and type $\mathfrak{p} \in[0,1],[\sigma]=\sigma_{k}$ for $\sigma \in\left(\sigma_{k}, \sigma_{k+1}\right], k=0,1, \ldots, m, \sigma_{0}=0$. The functions $f, g: J \times \mathbb{R} \times \mathbb{R} \longrightarrow \mathbb{R}$ and $Z_{k}: \mathbb{R} \longrightarrow \mathbb{R}$, $k=1,2, \ldots, m$, are continuous functions fulfilling some conditions that will be described later. Further, $w_{1}, w_{2} \in \mathbb{R}, \sigma_{k}$ satisfy $0=\sigma_{0}<\sigma_{1}<\cdots<\sigma_{k}<\sigma_{k+1}=\sigma,\left.\Delta \mathfrak{u}\right|_{\sigma=\sigma_{k}}=\mathfrak{u}\left(\sigma_{k}^{+}\right)-$ $\mathfrak{u}\left(\sigma_{k}^{-}\right)=\mathfrak{u}\left(\sigma_{k}^{+}\right)-\mathfrak{u}\left(\sigma_{k}\right), \mathfrak{u}\left(\sigma_{k}^{+}\right)=\lim _{h \rightarrow 0^{+}} \mathfrak{u}\left(\sigma_{k}+h\right), \mathfrak{u}\left(\sigma_{k}^{-}\right)=\lim _{h \rightarrow 0^{-}} \mathfrak{u}\left(\sigma_{k}+h\right)$ represent the right and left limits of $\mathfrak{u}(\sigma)$ at $\sigma \in\left(\sigma_{k}, \sigma_{k+1}\right], k=0,1, \ldots, m, .\left.\Delta \vartheta\right|_{\sigma=\sigma_{k}}=\vartheta\left(\sigma_{k}^{+}\right)-\vartheta\left(\sigma_{k}^{-}\right)=$ $\vartheta\left(\sigma_{k}^{+}\right)-\vartheta\left(\sigma_{k}\right), \vartheta\left(\sigma_{k}^{+}\right)=\lim _{h \rightarrow 0^{+}} \vartheta\left(\sigma_{k}+h\right)$ and $\vartheta\left(\sigma_{k}^{-}\right)=\lim _{h \rightarrow 0^{-}} \vartheta\left(\sigma_{k}+h\right)$ represent the right and left limits of $\vartheta(\sigma)$ at $\sigma \in\left(\sigma_{k}, \sigma_{k+1}\right], k=0,1, \ldots, m$.

The coupled systems of $\varphi$-Hilfer FDEs with impulsive conditions considered in this work are a wider class of coupled systems of BVPs that incorporates the BVPs for FDEs involving the most broadly used Riemann-Liouville and Caputo fractional derivatives. Regardless of this, the coupled systems (1.1) for various values of a function $\varphi$ and parameter $\mathfrak{p}$ include coupled systems of FDEs involving the Hilfer, Hadamard, Katugampola, and many other fractional derivative operators.

- If $\varphi(\sigma)=\sigma$ and $\mathfrak{p}=1$, then system (1.1) reduces to an implicit impulsive coupled system with the Caputo fractional derivative.

- If $\varphi(\sigma)=\sigma$ and $\mathfrak{p}=0$, then system (1.1) reduces to an implicit impulsive coupled system with the Riemann-Liouville fractional derivative.

- If $\mathfrak{p}=0$, then system (1.1) reduces to an implicit impulsive coupled system with the $\varphi$-Riemann-Liouville fractional derivative.

- If $\varphi(\sigma)=\sigma$, then system (1.1) reduces to an implicit impulsive coupled system with the Hilfer fractional derivative.

- If $\varphi(\sigma)=\log \sigma$, then system (1.1) reduces to an implicit impulsive coupled system with the Hilfer-Hadamard fractional derivative.

- If $\varphi(\sigma)=\sigma^{\rho}$, then system (1.1) reduces to an implicit impulsive coupled system with the Katugampola fractional derivative. 
The major contribution of this paper is obtaining an equivalent fractional integral equation of the proposed system and establishing the existence, uniqueness, and UH and GUH stability of a solution for an implicit impulsive coupled system with $\varphi$-Hilfer FD. Our analysis relies on the Banach and Leray-Schauder fixed point theorems. Though we use the standard methodology to obtain our results, its exposition to the proposed system is new. The acquired results obtained in this paper are more general and cover many parallel problems that contain particular cases of functions because our proposed system contains a global fractional derivative that integrates many classic fractional derivatives. Moreover, the results obtained in this work can be extended to $n$-tuple fractional systems (FSs). Our results include the results of Almalahi et al. [15], Abdo et al. [6], and Kharade et al. [35] and will be a useful contribution to the existing literature on this topic.

This paper is organized as follows. In Sect. 2, we render the rudimentary definitions and prove some lemmas and present some concepts of fixed point theorems. In Sect. 3, we prove the existence and uniqueness of solutions for impulsive implicit coupled system (1.1). In Sect. 4, we discuss the stability by means of mathematical analysis techniques. In Sect. 5, we give a pertinent example illustrating our results. Concluding remarks are presented in the last section.

\section{Background material and auxiliary results}

In this part, we give important definitions and auxiliary lemmas pertinent to our main results.

Let $J:=[0, T]$ and $J^{\prime}:=(0, T]$. Let $\mathbb{R}=\mathcal{C}(J)$ be the Banach space of continuous functions $\mathfrak{u}: J^{\prime} \rightarrow \mathbb{R}$ with the norm $\|\mathfrak{u}\|=\max \{|\mathfrak{u}(\sigma)|: \sigma \in J\}$. Clearly, $\mathbb{R}$ is a Banach space with this norm, and hence the product space $\mathbb{R} \times \mathbb{R}$ is also a Banach space with the norm

$$
\|(\mathfrak{u}, \vartheta)\|=\|\mathfrak{u}\|+\|\vartheta\| .
$$

We define the space $\mathcal{P C}(J)$ of piecewise continuous functions $\mathfrak{u}: J^{\prime} \rightarrow \mathbb{R}$ by

$$
\mathcal{P C}(J)=\left\{\begin{array}{c}
\mathfrak{u}: J^{\prime} \rightarrow \mathbb{R} ; \mathfrak{u}(\sigma) \in \mathcal{C}\left(\left(\sigma_{k}, \sigma_{k+1}\right], \mathbb{R}\right) ; k=0,1, \ldots, m, \\
\mathfrak{u}\left(\sigma_{k}^{+}\right) \text {and } \mathfrak{u}\left(\sigma_{k}^{-}\right) \text {exist with } \mathfrak{u}\left(\sigma_{k}^{+}\right)=\mathfrak{u}\left(\sigma_{k}^{-}\right) \text {for } k=0,1, \ldots, m
\end{array}\right\} .
$$

Obviously, $\mathcal{P C}(J)$ is a Banach space endowed with the norm

$$
\|\mathfrak{u}\|_{\mathcal{P C}(J)}=\max _{\sigma \in J}|\mathfrak{u}(\sigma)|
$$

Define the product space $\mathcal{B}=\mathcal{P C}(J) \times \mathcal{P C}(J)$ with the norm

$$
\|(\mathfrak{u}, \vartheta)\|_{\mathcal{B}}=\|\mathfrak{u}\|_{\mathcal{P C}())}+\|\vartheta\|_{\mathcal{P C}()}
$$

for $(\mathfrak{u}, \vartheta) \in \mathcal{B}$.

Definition 2.1 ([36]) Let $\mathfrak{y}>0$ and $f \in L_{1}(J)$. Then the generalized RL fractional integral of a function $f$ of order $\mathfrak{y}$ with respect to $\varphi$ is defined as

$$
\mathcal{I}_{0^{+}}^{\mathfrak{y}, \varphi} f(\sigma)=\frac{1}{\Gamma(\mathfrak{y})} \int_{0}^{\sigma} \varphi^{\prime}(s)(\varphi(\sigma)-\varphi(s))^{\mathfrak{y}-1} f(s) d s .
$$


Definition 2.2 ([41]) Let $n-1<\mathfrak{y}<n \in \mathbb{N}$, and let $f, \varphi \in \mathcal{P C}(J)$. Then the generalized Hilfer fractional derivative of a function $f$ of order $\mathfrak{y}$ and type $0 \leq \mathfrak{p} \leq 1$ with respect to $\varphi$ is defined as

$$
\begin{aligned}
{ }^{H} \mathcal{D}_{0^{+}}^{\mathfrak{y}, \mathfrak{p}, \varphi} f(\sigma) & =\mathcal{I}_{0^{+}}^{\mathfrak{p}(n-\mathfrak{y}) ; \varphi} f_{\varphi}^{[n]} \mathcal{I}_{0^{+}}^{(1-\mathfrak{p})(n-\mathfrak{y}), \varphi} f(\sigma) \\
& =\mathcal{I}_{0^{+}}^{\mathfrak{p}(n-\mathfrak{y}) ; \varphi} f_{\varphi}^{[n]} \mathcal{I}_{0^{+}}^{n-\gamma, \varphi} f(\sigma) \\
& =\mathcal{I}_{0^{+}}^{\mathfrak{p}(n-\mathfrak{y}) ; \varphi} \mathcal{D}_{a^{+}}^{\gamma ; \varphi} f(\sigma), \quad \gamma=\mathfrak{y}+n \mathfrak{p}-\mathfrak{y} \mathfrak{p},
\end{aligned}
$$

where

$$
\mathcal{D}_{0^{+}}^{\gamma ; \varphi} f(\sigma)=f_{\varphi}^{[n]} \mathcal{I}_{0^{+}}^{(1-\mathfrak{p})(n-\mathfrak{y}) ; \varphi} f(\sigma), \quad \text { and } \quad f_{\varphi}^{[n]}=\left(\frac{1}{\varphi^{\prime}(\sigma)} \frac{d}{d \sigma}\right)^{n} .
$$

Lemma 2.3 ([41] ) Let $\gamma=\mathfrak{y}+\mathfrak{p}-\mathfrak{y p}, \mathfrak{y}>0, \mathfrak{p}>0$, and $u \in \mathcal{P C}_{1-\gamma ; \varphi}^{\gamma}(J)$. Then

$$
\mathcal{I}_{0^{+}}^{\gamma ; \varphi} \mathcal{D}_{0^{+}}^{\gamma ; \varphi} u=\mathcal{I}_{0^{+}}^{\mathfrak{y} ; \varphi} \mathcal{D}_{0^{+}}^{\mathfrak{y}, \mathfrak{p} ; \varphi} u \text { and } \quad \mathcal{D}_{0^{+}}^{\gamma ; \varphi} \mathcal{I}_{0^{+}}^{\mathfrak{y} ; \varphi} u=\mathcal{D}_{0^{+}}^{\mathfrak{p}(1-\mathfrak{y}) ; \varphi} u
$$

Theorem 2.4 ([41] ) Let $0 \leq \gamma<\mathfrak{y}$ and $u \in \mathcal{P C}(J)$. Then $\mathcal{I}_{0^{+}}^{\mathfrak{y} ; \varphi} u(0)=\lim _{\sigma \rightarrow 0^{+}} I_{0^{+}}^{\mathfrak{y} ; \varphi} u(\sigma)=0$.

Lemma $2.5([36,41])$ Let $\mathfrak{y}, \mathfrak{p}>0$ and $\delta>0$. Then

$$
\begin{aligned}
& \mathcal{I}_{0^{+}}^{\mathfrak{y}, \varphi} \mathcal{I}_{0^{+}}^{\mathfrak{p}, \varphi} f(\sigma)=\mathcal{I}_{0^{+}}^{\mathfrak{y}+\mathfrak{p}, \varphi} f(\sigma), \\
& \mathcal{I}_{0^{+}}^{\mathfrak{y}, \varphi}(\varphi(\sigma)-\varphi(0))^{\delta-1}=\frac{\Gamma(\gamma)}{\Gamma(\mathfrak{y}+\gamma)}(\varphi(\sigma)-\varphi(0))^{\mathfrak{y}+\delta-1},
\end{aligned}
$$

and

$$
{ }^{H} \mathcal{D}_{0^{+}}^{\mathfrak{y}, \mathfrak{p}, \varphi}(\varphi(\sigma)-\varphi(0))^{\gamma-1}=0, \quad \gamma=\mathfrak{y}+n \mathfrak{p}-\mathfrak{y p} .
$$

Lemma 2.6 ([41]) If $\in \mathcal{P C}^{n}(J), n-1<\mathfrak{y}<n$, and $0 \leq \mathfrak{p} \leq 1$, then

$$
\mathcal{I}_{0^{+}}^{\mathfrak{y} ; \varphi}{ }^{H} \mathcal{D}_{0^{+}}^{\mathfrak{y}, \mathfrak{p}, \varphi} f(\sigma)=f(\sigma)-\sum_{k=1}^{n} \frac{(\varphi(\sigma)-\varphi(0))^{\gamma-k}}{\Gamma(\gamma-k+1)} f_{\varphi}^{[n-k]} \mathcal{I}_{a^{+}}^{(1-\mathfrak{p})(n-\mathfrak{y}) ; \varphi} f(0)
$$

and

$$
{ }^{H} \mathcal{D}_{0^{+}}^{\mathfrak{y}, \mathfrak{p}, \varphi} \mathcal{I}_{0^{+}}^{\mathfrak{y} ; \varphi} f(\sigma)=f(\sigma) .
$$

Lemma 2.7 ([31] (Leray-Schauder alternative)) Let $\Xi: \mathcal{X} \rightarrow \mathcal{X}$ be a completely continuous operator, and let $\digamma(\Xi)=\{y \in \mathcal{X}: y=\xi \Xi(y), \xi \in[0,1]\}$. Then either the set $\digamma(\Xi)$ is unbounded, or $\Xi$ has at least one fixed point.

Theorem 2.8 ([29] (Banach fixed point theorem)) Let $\mathcal{X}$ be a Banach space, let $K \subset \mathcal{X}$ be closed, and let $\Xi: K \rightarrow K$ be a strict contraction, that is, $\|\Xi(x)-\Xi(y)\| \leq L\|x-y\|$ for some $0<L<1$ and all $x, y \in K$. Then $\Xi$ has a fixed point in $K$. 
Lemma 2.9 Let $\gamma=\mathfrak{y}+\mathfrak{p}-\mathfrak{y p}, \mathfrak{y} \in(0,1), \mathfrak{p} \in[0,1]$, and let $\varpi: J^{\prime} \rightarrow \mathbb{R}$ be a continuous function. Then $\mathfrak{u} \in \mathcal{P C}^{\gamma}(J)$ satisfies

$$
\begin{cases}\mathcal{D}_{[\sigma]}^{\mathfrak{y}, \mathfrak{p}, \varphi} \mathfrak{u}(\sigma)=\varpi(\sigma), & \sigma \in J:=[0, T], \sigma \neq \sigma_{k}, k=1, \ldots, m, \\ \left.\Delta \mathfrak{u}\right|_{\sigma=\sigma_{k}}=Z_{k} \mathfrak{u}\left(\sigma_{k}^{-}\right), & k=1, \ldots, m, \\ \mathfrak{u}(T)=w & \end{cases}
$$

if and only if $\mathfrak{u}$ satisfies the following integral equations:

$$
\mathfrak{u}(\sigma)=\left\{\begin{array}{l}
\frac{(\varphi(\sigma)-\varphi(0))^{\gamma-1}}{(\varphi(T)-\varphi(0))^{\gamma-1}}\left[w-\mathcal{I}_{0^{+}}^{\mathfrak{y}, \varphi} \varpi(s)(T)\right]+\mathcal{I}_{0^{+}}^{\mathfrak{y}, \varphi} \varpi(s)(\sigma), \quad \sigma \in\left[0, \sigma_{1}\right], \\
\sum_{i=1}^{k+1} \frac{\left(\varphi\left(\sigma_{i}\right)-\varphi\left(\sigma_{i-1}\right)\right)^{\gamma-1}}{\left(\varphi(T)-\varphi\left(\sigma_{i-1}\right)\right)^{\gamma-1}}\left[w-\mathcal{I}_{i-1^{+}}^{\mathfrak{y}, \varphi} \varpi(s)(T)\right]+\sum_{i=1}^{k} \mathcal{I}_{\sigma_{i-1}^{\mathfrak{y}}, \varphi} \varpi(s)\left(\sigma_{i}\right) \\
\quad+\mathcal{I}_{\sigma_{k}^{+}, \varphi}^{\mathfrak{y}, \varphi}(s)(\sigma)+\sum_{i=1}^{k} Z_{i} \mathfrak{u}\left(\sigma_{i}^{-}\right), \quad \sigma \in\left(\sigma_{k}, \sigma_{k+1}\right], k=1, \ldots, m .
\end{array}\right.
$$

Proof First, let $\mathfrak{u} \in \mathcal{P C}^{\gamma}(J)$ be a solution of problem (2.1). We prove that $\mathfrak{u}$ is a solution of (2.2).

If $\sigma \in\left[0, \sigma_{1}\right]$, then $\mathcal{D}_{[\sigma]}^{\mathfrak{y}, \mathfrak{p}, \varphi} \mathfrak{u}(\sigma)=\varpi(\sigma),[\sigma]=0$. Taking the operator $\mathcal{I}_{0^{+}}^{\mathfrak{y}, \varphi}$ on both sides of the first equation in (2.1) and using Lemma 2.6, we have

$$
\mathfrak{u}(\sigma)=\frac{(\varphi(\sigma)-\varphi(0))^{\gamma-1}}{\Gamma(\gamma)} \mathcal{I}_{0^{+}}^{1-\gamma, \varphi} \mathfrak{u}(0)+\mathcal{I}_{0^{+}}^{\mathfrak{y}, \varphi} \varpi(s)(\sigma) .
$$

By the terminal condition we have

$$
\mathcal{I}_{0^{+}}^{1-\gamma, \varphi} \mathfrak{u}(0)=\frac{\Gamma(\gamma)}{(\varphi(T)-\varphi(0))^{\gamma-1}}\left[w-\mathcal{I}_{0^{+}}^{\mathfrak{y}, \varphi} \varpi(s)(T)\right] .
$$

Putting (2.4) into (2.3), we get

$$
\mathfrak{u}(\sigma)=\frac{(\varphi(\sigma)-\varphi(0))^{\gamma-1}}{(\varphi(T)-\varphi(0))^{\gamma-1}}\left[w-\mathcal{I}_{0^{+}}^{\mathfrak{y}, \varphi} \varpi(s)(T)\right]+\mathcal{I}_{0^{+}}^{\mathfrak{y}, \varphi} \varpi(s)(\sigma) .
$$

This means

$$
\mathfrak{u}\left(\sigma_{1}^{-}\right)=\frac{\left(\varphi\left(\sigma_{1}\right)-\varphi(0)\right)^{\gamma-1}}{(\varphi(T)-\varphi(0))^{\gamma-1}}\left[w-\mathcal{I}_{0^{+}}^{\mathfrak{y}, \varphi} \varpi(s)(T)\right]+\mathcal{I}_{0^{+}}^{\mathfrak{y}, \varphi} \varpi(s)\left(\sigma_{1}\right) .
$$

Since $\mathfrak{u}\left(\sigma_{1}^{-}\right)=\mathfrak{u}\left(\sigma_{1}^{+}\right)-Z_{1} \mathfrak{u}\left(\sigma_{1}^{-}\right)$, we get

$$
\mathfrak{u}\left(\sigma_{1}^{+}\right)=\frac{\left(\varphi\left(\sigma_{1}\right)-\varphi(0)\right)^{\gamma-1}}{(\varphi(T)-\varphi(0))^{\gamma-1}}\left[w-\mathcal{I}_{0^{+}}^{\mathfrak{y}, \varphi} \varpi(s)(T)\right]+\mathcal{I}_{0^{+}}^{\mathfrak{y}, \varphi} \varpi(s)\left(\sigma_{1}\right)+Z_{1} \mathfrak{u}\left(\sigma_{1}^{-}\right) .
$$

If $\sigma \in\left(\sigma_{1}, \sigma_{2}\right]$, then $\mathcal{D}_{[\sigma]}^{\mathfrak{y}, \mathfrak{p}, \varphi} \mathfrak{u}(\sigma)=\varpi(\sigma),[\sigma]=\sigma_{1}$, and $\mathfrak{u}(\sigma)$ is given by

$$
\begin{aligned}
\mathfrak{u}(\sigma)= & \mathfrak{u}\left(\sigma_{1}^{+}\right)+\frac{\left(\varphi(\sigma)-\varphi\left(\sigma_{1}\right)\right)^{\gamma-1}}{\left(\varphi(T)-\varphi\left(\sigma_{1}\right)\right)^{\gamma-1}}\left[w-\mathcal{I}_{\sigma_{1}^{+}}^{\mathfrak{y}, \varphi} \varpi(s)(T)\right]+\mathcal{I}_{\sigma_{1}^{+}}^{\mathfrak{y}, \varphi} \varpi(s)(\sigma) \\
= & \frac{\left(\varphi\left(\sigma_{1}\right)-\varphi(0)\right)^{\gamma-1}}{(\varphi(T)-\varphi(0))^{\gamma-1}}\left[w-\mathcal{I}_{0^{+}}^{\mathfrak{y}, \varphi} \varpi(s)(T)\right]+\frac{\left(\varphi(\sigma)-\varphi\left(\sigma_{1}\right)\right)^{\gamma-1}}{\left(\varphi(T)-\varphi\left(\sigma_{1}\right)\right)^{\gamma-1}}\left[w-\mathcal{I}_{\sigma_{1}^{+}}^{\mathfrak{y}, \varphi} \varpi(s)(T)\right] \\
& +\mathcal{I}_{0^{+}}^{\mathfrak{y}, \varphi} \varpi(s)\left(\sigma_{1}\right)+\mathcal{I}_{\sigma_{1}^{+}, \varphi}^{\mathfrak{y}, \varphi}(s)(\sigma)+Z_{1} \mathfrak{u}\left(\sigma_{1}^{-}\right) .
\end{aligned}
$$


This means that

$$
\begin{aligned}
\mathfrak{u}\left(\sigma_{2}^{-}\right)= & \frac{\left(\varphi\left(\sigma_{1}\right)-\varphi(0)\right)^{\gamma-1}}{(\varphi(T)-\varphi(0))^{\gamma-1}}\left[w-\mathcal{I}_{0^{+}}^{\mathfrak{y}, \varphi} \varpi(s)(T)\right] \\
& +\frac{\left(\varphi\left(\sigma_{2}\right)-\varphi\left(\sigma_{1}\right)\right)^{\gamma-1}}{\left(\varphi(T)-\varphi\left(\sigma_{1}\right)\right)^{\gamma-1}}\left[w-\mathcal{I}_{\sigma_{1}^{+}}^{\mathfrak{y}, \varphi} \varpi(s)(T)\right] \\
& +\mathcal{I}_{0^{+}}^{\mathfrak{y}, \varphi} \varpi(s)\left(\sigma_{1}\right)+\mathcal{I}_{\sigma_{1}^{+}}^{\mathfrak{y}, \varphi} \varpi(s)\left(\sigma_{2}\right)+Z_{1} \mathfrak{u}\left(\sigma_{1}^{-}\right) .
\end{aligned}
$$

Since $\mathfrak{u}\left(\sigma_{2}^{-}\right)=\mathfrak{u}\left(\sigma_{2}^{+}\right)-Z_{2} \mathfrak{u}\left(\sigma_{2}^{-}\right)$, we get

$$
\begin{aligned}
\mathfrak{u}\left(\sigma_{2}^{+}\right)= & \frac{\left(\varphi\left(\sigma_{1}\right)-\varphi(0)\right)^{\gamma-1}}{(\varphi(T)-\varphi(0))^{\gamma-1}}\left[w-\mathcal{I}_{0^{+}}^{\mathfrak{y}, \varphi} \varpi(s)(T)\right] \\
& +\frac{\left(\varphi\left(\sigma_{2}\right)-\varphi\left(\sigma_{1}\right)\right)^{\gamma-1}}{\left(\varphi(T)-\varphi\left(\sigma_{1}\right)\right)^{\gamma-1}}\left[w-\mathcal{I}_{\sigma_{1}^{\mathfrak{y}}, \varphi}^{\mathfrak{n}} \varpi(s)(T)\right] \\
& +\mathcal{I}_{0^{+}}^{\mathfrak{y}, \varphi} \varpi(s)\left(\sigma_{1}\right)+\mathcal{I}_{\sigma_{1}^{+}}^{\mathfrak{y}, \varphi} \varpi(s)\left(\sigma_{2}\right)+Z_{1} \mathfrak{u}\left(\sigma_{1}^{-}\right)+Z_{2} \mathfrak{u}\left(\sigma_{2}^{-}\right) .
\end{aligned}
$$

If $\sigma \in\left(\sigma_{2}, \sigma_{3}\right]$, then $\mathcal{D}_{[\sigma]}^{\mathfrak{y}, \mathfrak{p}, \varphi} \mathfrak{u}(\sigma)=\varpi(\sigma),[\sigma]=\sigma_{2}$, and $\mathfrak{u}(\sigma)$ is given by

$$
\begin{aligned}
& \mathfrak{u}(\sigma)=\mathfrak{u}\left(\sigma_{2}^{+}\right)+\frac{\left(\varphi(\sigma)-\varphi\left(\sigma_{2}\right)\right)^{\gamma-1}}{\left(\varphi(T)-\varphi\left(\sigma_{2}\right)\right)^{\gamma-1}}\left[w-\mathcal{I}_{\sigma_{2}^{+}}^{\mathfrak{y}, \varphi} \varpi(s)(T)\right]+\mathcal{I}_{\sigma_{2}^{+}}^{\mathfrak{y}, \varphi} \varpi(s)(\sigma) \\
& =\frac{\left(\varphi\left(\sigma_{1}\right)-\varphi(0)\right)^{\gamma-1}}{(\varphi(T)-\varphi(0))^{\gamma-1}}\left[w-\mathcal{I}_{0^{+}}^{\mathfrak{y}, \varphi} \varpi(s)(T)\right] \\
& +\frac{\left(\varphi\left(\sigma_{2}\right)-\varphi\left(\sigma_{1}\right)\right)^{\gamma-1}}{\left(\varphi(T)-\varphi\left(\sigma_{1}\right)\right)^{\gamma-1}}\left[w-\mathcal{I}_{\sigma_{1}^{+}}^{\mathfrak{y}, \varphi} \varpi(s)(T)\right] \\
& +\frac{\left(\varphi(\sigma)-\varphi\left(\sigma_{2}\right)\right)^{\gamma-1}}{\left(\varphi(T)-\varphi\left(\sigma_{2}\right)\right)^{1 \gamma-1}}\left[w-\mathcal{I}_{\sigma_{2}^{+}}^{\mathfrak{y}, \varphi} \varpi(s)(T)\right] \\
& +\mathcal{I}_{0^{+}}^{\mathfrak{y}, \varphi} \varpi(s)\left(\sigma_{1}\right)+\mathcal{I}_{\sigma_{1}^{+}}^{\mathfrak{y}, \varphi} \varpi(s)\left(\sigma_{2}\right)+\mathcal{I}_{\sigma_{2}^{+}}^{\mathfrak{y}, \varphi} \varpi(s)(\sigma)+Z_{1} \mathfrak{u}\left(\sigma_{1}^{-}\right)+Z_{2} \mathfrak{u}\left(\sigma_{2}^{-}\right) .
\end{aligned}
$$

This means that

$$
\begin{aligned}
\mathfrak{u}\left(\sigma_{3}^{-}\right)= & \frac{\left(\varphi\left(\sigma_{1}\right)-\varphi(0)\right)^{\gamma-1}}{(\varphi(T)-\varphi(0))^{\gamma-1}}\left[w-\mathcal{I}_{0^{+}}^{\mathfrak{y}, \varphi} \varpi(s)(T)\right] \\
& +\frac{\left(\varphi\left(\sigma_{2}\right)-\varphi\left(\sigma_{1}\right)\right)^{\gamma-1}}{\left(\varphi(T)-\varphi\left(\sigma_{1}\right)\right)^{\gamma-1}}\left[w-\mathcal{I}_{\sigma_{1}^{+}, \varphi}^{\mathfrak{y}} \varpi(s)(T)\right] \\
& +\frac{\left(\varphi\left(\sigma_{3}\right)-\varphi\left(\sigma_{2}\right)\right)^{\gamma-1}}{\left(\varphi(T)-\varphi\left(\sigma_{2}\right)\right)^{\gamma-1}}\left[w-\mathcal{I}_{\sigma_{2}^{+}, \varphi}^{\mathfrak{y}} \varpi(s)(T)\right] \\
& +\mathcal{I}_{0^{+}, \varphi}^{\mathfrak{y}, \varphi}(s)\left(\sigma_{1}\right)+\mathcal{I}_{\sigma_{1}^{+}, \varphi}^{\mathfrak{y}} \varpi(s)\left(\sigma_{2}\right)+\mathcal{I}_{\sigma_{2}^{+}}^{\mathfrak{y}, \varphi} \varpi(s)\left(\sigma_{3}\right) \\
& +Z_{1} \mathfrak{u}\left(\sigma_{1}^{-}\right)+Z_{2} \mathfrak{u}\left(\sigma_{2}^{-}\right) .
\end{aligned}
$$

After impulse $\left(\mathfrak{u}\left(\sigma_{3}^{-}\right)=\mathfrak{u}\left(\sigma_{3}^{+}\right)-Z_{3} \mathfrak{u}\left(\sigma_{3}^{-}\right)\right)$, we get

$$
\begin{aligned}
\mathfrak{u}\left(\sigma_{3}^{-}\right)= & \frac{\left(\varphi\left(\sigma_{1}\right)-\varphi(0)\right)^{\gamma-1}}{(\varphi(T)-\varphi(0))^{\gamma-1}}\left[w-\mathcal{I}_{0^{+}}^{\mathfrak{y}, \varphi} \varpi(s)(T)\right] \\
& +\frac{\left(\varphi\left(\sigma_{2}\right)-\varphi\left(\sigma_{1}\right)\right)^{\gamma-1}}{\left(\varphi(T)-\varphi\left(\sigma_{1}\right)\right)^{\gamma-1}}\left[w-\mathcal{I}_{\sigma_{1}^{+}, \varphi}^{\mathfrak{y}} \varpi(s)(T)\right]
\end{aligned}
$$




$$
\begin{aligned}
& +\frac{\left(\varphi\left(\sigma_{3}\right)-\varphi\left(\sigma_{2}\right)\right)^{\gamma-1}}{\left(\varphi(T)-\varphi\left(\sigma_{2}\right)\right)^{\gamma-1}}\left[w-\mathcal{I}_{\sigma_{2}^{+}}^{\mathfrak{y}, \varphi} \varpi(s)(T)\right] \\
& +\mathcal{I}_{0^{+}}^{\mathfrak{y}, \varphi} \varpi(s)\left(\sigma_{1}\right)+\mathcal{I}_{\sigma_{1}^{+}, \varphi} \varpi(s)\left(\sigma_{2}\right)+\mathcal{I}_{\sigma_{2}^{+}}^{\mathfrak{y}, \varphi} \varpi(s)\left(\sigma_{3}\right) \\
& +Z_{1} \mathfrak{u}\left(\sigma_{1}^{-}\right)+Z_{2} \mathfrak{u}\left(\sigma_{2}^{-}\right)+Z_{3} \mathfrak{u}\left(\sigma_{3}^{-}\right) .
\end{aligned}
$$

If $\sigma \in\left(\sigma_{3}, \sigma_{4}\right]$, then $\mathcal{D}_{[\sigma]}^{\mathfrak{y}, \mathfrak{p}, \varphi} \mathfrak{u}(\sigma)=\varpi(\sigma),[\sigma]=\sigma_{3}$, and $\mathfrak{u}(\sigma)$ is given by

$$
\begin{aligned}
& \mathfrak{u}(\sigma)=\mathfrak{u}\left(\sigma_{3}^{+}\right)+\frac{\left(\varphi(\sigma)-\varphi\left(\sigma_{3}\right)\right)^{\gamma-1}}{\left(\varphi(T)-\varphi\left(\sigma_{3}\right)\right)^{\gamma-1}}\left[w-\mathcal{I}_{\sigma_{3}^{+}}^{\mathfrak{y}, \varphi} \varpi(s)(T)\right]+\mathcal{I}_{\sigma_{3}^{+}}^{\mathfrak{y}, \varphi} \varpi(s)(\sigma) \\
& =\frac{\left(\varphi\left(\sigma_{1}\right)-\varphi(0)\right)^{\gamma-1}}{(\varphi(T)-\varphi(0))^{\gamma-1}}\left[w-\mathcal{I}_{0^{+}}^{\mathfrak{n}, \varphi} \varpi(s)(T)\right] \\
& +\frac{\left(\varphi\left(\sigma_{2}\right)-\varphi\left(\sigma_{1}\right)\right)^{\gamma-1}}{\left(\varphi(T)-\varphi\left(\sigma_{1}\right)\right)^{\gamma-1}}\left[w-\mathcal{I}_{\sigma_{1}^{+}}^{\mathfrak{y}, \varphi} \varpi(s)(T)\right] \\
& +\frac{\left(\varphi\left(\sigma_{3}\right)-\varphi\left(\sigma_{2}\right)\right)^{\gamma-1}}{\left(\varphi(T)-\varphi\left(\sigma_{2}\right)\right)^{\gamma-1}}\left[w-\mathcal{I}_{\sigma_{2}^{+}}^{\mathfrak{y}, \varphi} \varpi(s)(T)\right] \\
& +\frac{\left(\varphi(\sigma)-\varphi\left(\sigma_{3}\right)\right)^{\gamma-1}}{\left(\varphi(T)-\varphi\left(\sigma_{3}\right)\right)^{\gamma-1}}\left[w-\mathcal{I}_{\sigma_{3}^{+}}^{\mathfrak{y}, \varphi} \varpi(s)(T)\right] \\
& +\mathcal{I}_{0^{+}}^{\mathfrak{y}, \varphi} \varpi(s)\left(\sigma_{1}\right)+\mathcal{I}_{\sigma_{1}^{+}}^{\mathfrak{y}, \varphi} \varpi(s)\left(\sigma_{2}\right)+\mathcal{I}_{\sigma_{2}^{+}}^{\mathfrak{y}, \varphi} \varpi(s)\left(\sigma_{3}\right)+\mathcal{I}_{\sigma_{3}^{+}}^{\mathfrak{y}, \varphi} \varpi(s)(\sigma) \\
& +Z_{1} \mathfrak{u}\left(\sigma_{1}^{-}\right)+Z_{2} \mathfrak{u}\left(\sigma_{2}^{-}\right)+Z_{3} \mathfrak{u}\left(\sigma_{3}^{-}\right) \text {. }
\end{aligned}
$$

Assume that

$$
\begin{aligned}
\mathfrak{u}\left(\sigma_{k}^{+}\right)= & \frac{\left(\varphi\left(\sigma_{1}\right)-\varphi(0)\right)^{\gamma-1}}{(\varphi(T)-\varphi(0))^{\gamma-1}}\left[w-\mathcal{I}_{0^{+}}^{\mathfrak{y}, \varphi} \varpi(s)(T)\right] \\
& +\frac{\left(\varphi\left(\sigma_{2}\right)-\varphi\left(\sigma_{1}\right)\right)^{\gamma-1}}{\left(\varphi(T)-\varphi\left(\sigma_{1}\right)\right)^{\gamma-1}}\left[w-\mathcal{I}_{\sigma_{1}^{+}}^{\mathfrak{y}, \varphi} \varpi(s)(T)\right] \\
& +\cdots+\frac{\left(\varphi\left(\sigma_{k}\right)-\varphi\left(\sigma_{k-1}\right)\right)^{\gamma-1}}{\left(\varphi(T)-\varphi\left(\sigma_{k-1}\right)\right)^{\gamma-1}}\left[w-\mathcal{I}_{\sigma_{k-1}^{\mathfrak{y}, \varphi}} \varpi(s)(T)\right] \\
& +\mathcal{I}_{0^{+}}^{\mathfrak{y}, \varphi} \varpi(s)\left(\sigma_{1}\right)+\mathcal{I}_{\sigma_{1}^{\mathfrak{y}}, \varphi} \varpi(s)\left(\sigma_{2}\right)+\cdots+\mathcal{I}_{\sigma_{k-1}^{\mathfrak{y}, \varphi}}^{+} \varpi(s)\left(\sigma_{k}\right) \\
& +Z_{1} \mathfrak{u}\left(\sigma_{1}^{-}\right)+Z_{2} \mathfrak{u}\left(\sigma_{2}^{-}\right)+\cdots+Z_{k} \mathfrak{u}\left(\sigma_{k}^{-}\right) .
\end{aligned}
$$

Then, inductively, for $\sigma \in\left(\sigma_{k}, \sigma_{k+1}\right]$, we have $\mathcal{D}_{[\sigma]}^{\mathfrak{y}, \mathfrak{p}, \varphi} \mathfrak{u}(\sigma)=\varpi(\sigma),[\sigma]=\sigma_{k}$, and $\mathfrak{u}(\sigma)$ is given by

$$
\begin{aligned}
\mathfrak{u}(\sigma)= & \mathfrak{u}\left(\sigma_{k}^{+}\right)+\frac{\left(\varphi(\sigma)-\varphi\left(\sigma_{k}\right)\right)^{\gamma-1}}{\left(\varphi(T)-\varphi\left(\sigma_{k}\right)\right)^{\gamma-1}}\left[w-\mathcal{I}_{\sigma_{k}^{+}}^{\mathfrak{y}, \varphi} \varpi(s)(T)\right]+\mathcal{I}_{\sigma_{k}^{+}}^{\mathfrak{y}, \varphi} \varpi(s)(\sigma) \\
= & \sum_{i=1}^{k+1} \frac{\left(\varphi\left(\sigma_{i}\right)-\varphi\left(\sigma_{i-1}\right)\right)^{\gamma-1}}{\left(\varphi(T)-\varphi\left(\sigma_{i-1}\right)\right)^{\gamma-1}}\left[w-\mathcal{I}_{i-1^{+}}^{\mathfrak{y}, \varphi} \varpi(s)(T)\right] \\
& +\sum_{i=1}^{k} \mathcal{I}_{\sigma_{i-1}^{\mathfrak{y}, \varphi}} \varpi(s)\left(\sigma_{i}\right)+\mathcal{I}_{\sigma_{k}^{+}}^{\mathfrak{y}, \varphi} \varpi(s)(\sigma)+\sum_{i=1}^{k} Z_{i} \mathfrak{u}\left(\sigma_{i}^{-}\right) .
\end{aligned}
$$

Thus (2.2) is satisfied.

Conversely, assume that $\mathfrak{u}$ satisfies equation (2.2). 
Case 1: $\sigma \in\left[0, \sigma_{1}\right]$.

Replacing $\sigma$ by $T$ in (2.2), we get $\mathfrak{u}(T)=w$. On the other hand, applying $\mathcal{D}_{0^{+}}^{\gamma ; \varphi}$ to both sides of (2.2) and using Lemma 2.3, we get

$$
\mathcal{D}_{0^{+}}^{\gamma ; \varphi} \mathfrak{u}(\sigma)=\mathcal{D}_{0^{+}}^{\mathfrak{p}(1-\mathfrak{y}) ; \varphi} \varpi(\sigma) .
$$

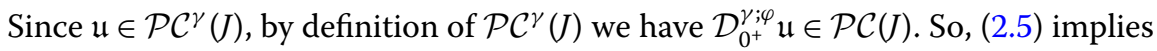

$$
\mathcal{D}_{0^{+}}^{\gamma ; \varphi} \mathfrak{u}(\sigma)=\mathcal{D}_{0^{+}}^{\mathfrak{p}(1-\mathfrak{y}) ; \varphi} \varpi(\sigma) \in \mathcal{P C}(J)
$$

For $\varpi \in \mathcal{P C}(J)$, it is obvious that $\mathcal{I}_{0^{+}}^{1-\mathfrak{p}(1-\mathfrak{y}) ; \varphi} \varpi \in \mathcal{P C}^{1}(J)$. Hence $\varpi$ and $\mathcal{I}_{0^{+}}^{1-\mathfrak{p}(1-\mathfrak{y}) ; \varphi} \varpi$ satisfy the conditions of Theorem 2.6. Now, applying $\mathcal{I}_{0^{+}}^{\mathfrak{p}(1-\mathfrak{y}) ; \varphi}$ to both sides of (2.5) and using Theorem 2.6, we get

$$
{ }^{H} \mathcal{D}_{0^{+}}^{\mathfrak{y}, \mathfrak{p} ; \varphi} \mathfrak{u}(\sigma)=\varpi(\sigma)-\frac{\mathcal{I}_{0^{+}}^{1-\mathfrak{p}(1-\mathfrak{y}) ; \varphi} \varpi(0)}{\Gamma(\mathfrak{p}(1-\mathfrak{y}))}(\varphi(\sigma)-\varphi(0))^{\mathfrak{p}(1-\mathfrak{y})-1}
$$

By Theorem 2.4 we have $\mathcal{I}_{0^{+}}^{1-\mathfrak{p}(1-\mathfrak{y}) ; \varphi} \varpi(0)=0$. Hence (2.6) becomes

$$
{ }^{H} \mathcal{D}_{0^{+}}^{\mathfrak{y}, \mathfrak{p} ; \varphi} \mathfrak{u}(\sigma)=\varpi(\sigma), \quad \sigma \in J
$$

Case 2: $\sigma \in\left(\sigma_{k}, \sigma_{k+1}\right]$.

By the same technique as in case 1 we can easily prove case 2 .

Lemma 2.10 Let $\gamma=\mathfrak{y}+\mathfrak{p}-\mathfrak{y p}$ be such that $\mathfrak{y} \in(0,1), \mathfrak{p} \in[0,1]$, and let $f, g: J^{\prime} \times \mathbb{R} \times \mathbb{R} \rightarrow \mathbb{R}$ be continuous functions. If $(\mathfrak{u}, \vartheta) \in \mathcal{B}$ satisfies problem (1.1), then by Lemma $2.9,(\mathfrak{u}, \vartheta)$ satisfies the following integral equations:

$$
\left\{\begin{array}{l}
\mathfrak{u}(\sigma)=\left\{\begin{array}{c}
\sum_{0<\sigma_{k}<\sigma} \frac{\left(\varphi\left(\sigma_{k}\right)-\varphi\left(\sigma_{k-1}\right)\right)^{\gamma-1}}{\left(\varphi(T)-\varphi\left(\sigma_{k-1}\right)\right)^{\gamma-1}}\left[w_{1}-\mathcal{I}_{\sigma_{k-1}^{+}, \varphi}^{\mathfrak{y}, \varphi} f\left(s, \mathfrak{u}(s), \mathcal{D}_{[\sigma]}^{\mathfrak{y}, \mathfrak{p}, \varphi} \vartheta(s)\right)(T)\right] \\
\quad+\sum_{0<\sigma_{k}<\sigma} \mathcal{I}_{\sigma_{k-1}^{+}, \varphi}^{+} f\left(s, \mathfrak{u}(s), \mathcal{D}_{[\sigma]}^{\mathfrak{y}, \mathfrak{p}, \varphi} \vartheta(s)\right)\left(\sigma_{k}\right)+\mathcal{I}_{\sigma_{k}^{+}}^{\mathfrak{y}, \varphi} f\left(s, \mathfrak{u}(s), \mathcal{D}_{[\sigma]}^{\mathfrak{y}, \mathfrak{p}, \varphi} \vartheta(s)\right)(\sigma) \\
\quad+\sum_{0<\sigma_{k}<\sigma} Z_{k} \mathfrak{u}\left(\sigma_{k}^{-}\right), \quad \sigma \in\left(\sigma_{k}, \sigma_{k+1}\right], k=1, \ldots, m,
\end{array}\right. \\
\vartheta(\sigma)=\left\{\begin{array}{c}
\sum_{0<\sigma_{k}<\sigma} \frac{\left(\varphi\left(\sigma_{k}\right)-\varphi\left(\sigma_{k-1}\right)\right)^{\gamma-1}}{\left(\varphi(T)-\varphi\left(\sigma_{k-1}\right)\right)^{\gamma-1}}\left[w_{2}-\mathcal{I}_{\sigma_{k-1}^{\mathfrak{y}, \varphi}}^{\mathfrak{y}, \varphi} g\left(s, \mathcal{D}_{[\sigma]}^{\mathfrak{y}, \mathfrak{p}, \varphi} \mathfrak{u}(s), \vartheta(s)\right)(T)\right] \\
\quad+\sum_{0<\sigma_{k}<\sigma} \mathcal{I}_{\sigma_{k-1}^{\mathfrak{y}, \varphi}} g\left(s, \mathcal{D}_{[\sigma]}^{\mathfrak{y}, \mathfrak{p}, \varphi} \mathfrak{u}(s), \vartheta(s)\right)\left(\sigma_{k}\right)+\mathcal{I}_{\sigma_{k}^{+}}^{\mathfrak{y}, \varphi} g\left(s, \mathcal{D}_{[\sigma]}^{\mathfrak{y}, \mathfrak{p}, \varphi} \mathfrak{u}(s), \vartheta(s)\right)(\sigma) \\
\quad+\sum_{0<\sigma_{k}<\sigma} Z_{k} \mathfrak{u}\left(\sigma_{k}^{-}\right), \quad \sigma \in\left(\sigma_{k}, \sigma_{k+1}\right], k=1, \ldots, m .
\end{array}\right.
\end{array}\right.
$$

Consider the continuous operator $\Xi: \mathcal{B} \rightarrow \mathcal{B}$ defined by

$$
\Xi(\mathfrak{u}, \vartheta)(\sigma)=\left(\Xi_{1}(\mathfrak{u}, \vartheta)(\sigma), \Xi_{2}(\vartheta, \mathfrak{u})(\sigma)\right)
$$

where

$$
\Xi_{1}(\mathfrak{u}, \vartheta)(\sigma)=\left\{\begin{aligned}
\sum_{0<\sigma_{k}<\sigma} \frac{\left(\varphi\left(\sigma_{k}\right)-\varphi\left(\sigma_{k-1}\right)\right)^{\gamma-1}}{\left(\varphi(T)-\varphi\left(\sigma_{k-1}\right)\right)^{\gamma-1}}\left[w_{1}-\mathcal{I}_{\sigma_{k-1}^{\mathfrak{y}}, \varphi}^{+} f\left(s, \mathfrak{u}(s), \mathcal{D}_{[\sigma]}^{\mathfrak{y}, \mathfrak{p}, \varphi} \vartheta(s)\right)(T)\right] \\
\quad+\sum_{0<\sigma_{k}<\sigma} \mathcal{I}_{\sigma_{k-1}^{\mathfrak{y}, \varphi}} f\left(s, \mathfrak{u}(s), \mathcal{D}_{0}^{\mathfrak{y}, \mathfrak{p}, \varphi} \vartheta(s)\right)\left(\sigma_{k}\right) \\
\quad+\mathcal{I}_{\sigma_{k}^{\mathfrak{y}}, \varphi}^{\mathfrak{y},} f\left(s, \mathfrak{u}(s), \mathcal{D}_{[\sigma]}^{\mathfrak{y}, \mathfrak{p}, \varphi} \vartheta(s)\right)(\sigma) \\
+\sum_{0<\sigma_{k}<\sigma} Z_{k} \mathfrak{u}\left(\sigma_{k}^{-}\right), \quad \sigma \in\left(\sigma_{k}, \sigma_{k+1}\right], k=1, \ldots, m,
\end{aligned}\right.
$$


and

$$
\Xi_{2}(\vartheta, \mathfrak{u})(\sigma)=\left\{\begin{aligned}
\sum_{0<\sigma_{k}<\sigma} \frac{\left(\varphi\left(\sigma_{k}\right)-\varphi\left(\sigma_{k-1}\right)\right)^{\gamma-1}}{\left(\varphi(T)-\varphi\left(\sigma_{k-1}\right)\right)^{\gamma-1}}\left[w_{2}-\mathcal{I}_{\sigma_{k-1}^{+}}^{\mathfrak{y}, \varphi} g\left(s, \mathcal{D}_{[\sigma]}^{\mathfrak{y}, \mathfrak{p}, \varphi} \mathfrak{u}(s), \vartheta(s)\right)(T)\right] \\
\quad+\sum_{0<\sigma_{k}<\sigma} \mathcal{I}_{\sigma_{k-1}^{\mathfrak{y}, \varphi}} g\left(s, \mathcal{D}_{[\sigma]}^{\mathfrak{y}, \mathfrak{p}, \varphi} \mathfrak{u}(s), \vartheta(s)\right)\left(\sigma_{k}\right) \\
\quad+\mathcal{I}_{\sigma_{k}^{+}}^{\mathfrak{y}, \varphi} g\left(s, \mathcal{D}_{[\sigma]}^{\mathfrak{y}, \mathfrak{p}, \varphi} \mathfrak{u}(s), \vartheta(s)\right)(\sigma) \\
+\sum_{0<\sigma_{k}<\sigma} Z_{k} \mathfrak{u}\left(\sigma_{k}^{-}\right), \quad \sigma \in\left(\sigma_{k}, \sigma_{k+1}\right], k=1, \ldots, m .
\end{aligned}\right.
$$

Note that the fixed points of the operator $\Xi$ are solutions of problem (1.1).

\section{Existence of solution}

In this section, we consider a general coupled system of Hilfer FDEs (1.1) involving an arbitrary function $\varphi$. To demonstrate our main results, we introduce the following hypotheses.

$\left(\mathrm{H}_{1}\right)$ The functions $f, g: J \times \mathbb{R} \times \mathbb{R} \rightarrow \mathbb{R}$ are continuous, and there exist constant numbers $\varrho_{f}, \varrho_{g}, \varrho_{f}^{\prime}, \varrho_{g}^{\prime}>0$ such that for all $(\mathfrak{u}, \vartheta),(\widehat{u}, \widehat{\vartheta}) \in \mathbb{R} \times \mathbb{R}$,

$$
\begin{aligned}
& |f(\sigma, \mathfrak{u}(\sigma), \vartheta(\sigma))-f(\sigma, \widehat{\mathfrak{u}}(\sigma), \widehat{\vartheta}(\sigma))| \leq \varrho_{f}|\mathfrak{u}(\sigma)-\widehat{\mathfrak{u}}(\sigma)|+\varrho_{f}^{\prime}|\vartheta(\sigma)-\widehat{\vartheta}(\sigma)|, \\
& |g(\sigma, \mathfrak{u}(\sigma), \vartheta(\sigma))-g(\sigma, \widehat{\mathfrak{u}}(\sigma), \widehat{\vartheta}(\sigma))| \leq \varrho_{g}|\mathfrak{u}(\sigma)-\widehat{\mathfrak{u}}(\sigma)|+\varrho_{g}^{\prime}|\vartheta(\sigma)-\widehat{\vartheta}(\sigma)| .
\end{aligned}
$$

$\left(\mathrm{H}_{2}\right)$ The functions $f, g: J \times \mathbb{R} \times \mathbb{R} \rightarrow \mathbb{R}$ are continuous functions such that for each $(\mathfrak{u}, \vartheta) \in \mathbb{R}$, there exist nondecreasing continuous linear functions $\omega_{f}, \omega_{g}: \mathbb{R}^{+} \rightarrow \mathbb{R}^{+}$such that

$$
\begin{aligned}
& |f(\sigma, \mathfrak{u}(\sigma), \vartheta(\sigma))| \leq \omega_{f}|\mathfrak{u}(\sigma)|+\omega_{f}^{\prime}|\vartheta(\sigma)| \\
& |g(\sigma, \mathfrak{u}(\sigma), \vartheta(\sigma))| \leq \omega_{g}|\mathfrak{u}(\sigma)|+\omega_{g}^{\prime}|\vartheta(\sigma)|
\end{aligned}
$$

$\left(\mathrm{H}_{3}\right)$ The functions $Z_{k}: \mathbb{R} \rightarrow \mathbb{R}$ are continuous, and there exists a constant $L_{Z}>0$ such that

$$
\left|Z_{k}(\Theta)-Z_{k}\left(\Theta^{*}\right)\right| \leq L_{Z}\left|\Theta-\Theta^{*}\right|, \quad k=1, \ldots, m, \Theta, \Theta^{*} \in \mathbb{R}
$$

In the following, we will apply the Theorem 2.7 to obtain an existence result for system (1.1).

Theorem 3.1 Assume that $\left(H_{1}\right)-\left(H_{3}\right)$ hold. If

$$
Q_{1}:=\frac{(2 m+1)\left[\omega_{f}\left(1+\omega_{g}\right)+\omega_{g}^{\prime}\left(1+\omega_{f}^{\prime}\right)\right]}{2 \Gamma(\mathfrak{y}+1)\left(1-\omega_{f}^{\prime} \omega_{g}\right)}(\varphi(T)-\varphi(0))^{\mathfrak{y}}+m L_{Z}<1,
$$

then problem (1.1) has at least one solution on J.

Proof Define the closed ball set

$$
\mathbb{B}_{R}=\left\{(\mathfrak{u}, \vartheta) \in \mathcal{B}:\|(\mathfrak{u}, \vartheta)\|_{\mathcal{P C}(J)} \leq R,\|\mathfrak{u}\|_{\mathcal{P C}(J)} \leq \frac{R}{2},\|\vartheta\|_{\mathcal{P C}(J)} \leq \frac{R}{2}\right\}
$$


with

$$
R \geq \frac{m\left[\left|w_{1}\right|+\left|w_{2}\right|\right]}{1-Q_{1}} .
$$

We will prove that the operator $\Xi$ defined by (2.7) has a fixed point by using Theorem 2.7 . For this, we divide the proof into three steps.

Step 1: $\Xi\left(\mathbb{B}_{R}\right) \subset \mathbb{B}_{R}$.

For any $(\mathfrak{u}, \vartheta) \in \mathbb{B}_{R}$, we have

$$
\|\Xi(\mathfrak{u}, \vartheta)\|_{\mathcal{B}} \leq\left\|\Xi_{1}(\mathfrak{u}, \vartheta)\right\|_{\mathcal{P C}())}+\left\|\Xi_{2}(\vartheta, \mathfrak{u})\right\|_{\mathcal{P C}())} .
$$

From equation (2.8) we have

$$
\begin{aligned}
& \left|\Xi_{1}(\mathfrak{u}, \vartheta)\right| \leq \sum_{0<\sigma_{k}<\sigma} \frac{\left(\varphi\left(\sigma_{k}\right)-\varphi\left(\sigma_{k-1}\right)\right)^{\gamma-1}}{\left(\varphi(T)-\varphi\left(\sigma_{k-1}\right)\right)^{\gamma-1}} \\
& {\left[\left|w_{1}\right|+\mathcal{I}_{\sigma_{k-1}^{+}, \varphi}^{\mathfrak{y}, \varphi}\left|f\left(s, \mathfrak{u}(s), \mathcal{D}_{[\sigma]}^{\mathfrak{y}, \mathfrak{p}, \varphi} \vartheta(s)\right)\right|(T)\right]} \\
& +\sum_{0<\sigma_{k}<\sigma} \mathcal{I}_{\sigma_{k-1}^{\mathfrak{y}}, \varphi}^{\mathfrak{p}}\left|f\left(s, \mathfrak{u}(s), \mathcal{D}_{0}^{\mathfrak{y}, \mathfrak{p}, \varphi} \vartheta(s)\right)\right|\left(\sigma_{k}\right) \\
& +\mathcal{I}_{\sigma_{k}^{+}}^{\mathfrak{y}, \varphi}\left|f\left(s, \mathfrak{u}(s), \mathcal{D}_{[\sigma]}^{\mathfrak{y}, \mathfrak{p}, \varphi} \vartheta(s)\right)\right|(\sigma) \\
& +\sum_{0<\sigma_{k}<\sigma}\left|Z_{k} \mathfrak{u}\left(\sigma_{k}^{-}\right)\right| \\
& \leq m\left|w_{1}\right|+m \mathcal{I}_{\sigma_{k-1}^{+}}^{\mathfrak{y}, \varphi}\left|f\left(s, \mathfrak{u}(s), \mathcal{D}_{[\sigma]}^{\mathfrak{y}, \mathfrak{p}, \varphi} \vartheta(s)\right)\right|(T) \\
& +m \mathcal{I}_{\sigma_{k-1}^{+}}^{\mathfrak{y}, \varphi}\left|f\left(s, \mathfrak{u}(s), \mathcal{D}_{[\sigma]}^{\mathfrak{y}, \mathfrak{p}, \varphi} \vartheta(s)\right)\right|\left(\sigma_{k}\right) \\
& +\mathcal{I}_{\sigma_{k}^{+}}^{\mathfrak{y}, \varphi}\left|f\left(s, \mathfrak{u}(s), \mathcal{D}_{[\sigma]}^{\mathfrak{y}, \mathfrak{p}, \varphi} \vartheta(s)\right)\right|(\sigma)+m L_{Z}|\mathfrak{u}(\sigma)| \\
& \leq m\left|w_{1}\right|+(2 m+1)\left[\frac{\omega_{f}\|\mathfrak{u}\|_{\mathcal{P C}())}+\omega_{f}^{\prime} \omega_{g}^{\prime}\|\vartheta\|_{\mathcal{P C}())}}{\Gamma(\mathfrak{y}+1)\left(1-\omega_{f}^{\prime} \omega_{g}\right)}\right](\varphi(T)-\varphi(0))^{\mathfrak{y}} \\
& +m L_{Z}\|\mathfrak{u}\|_{\mathcal{P C}(J)} \\
& \leq m\left|w_{1}\right|+(2 m+1)\left[\frac{\left(\omega_{f}+\omega_{f}^{\prime} \omega_{g}^{\prime}\right) R}{2 \Gamma(\mathfrak{y}+1)\left(1-\omega_{f}^{\prime} \omega_{g}\right)}\right](\varphi(T)-\varphi(0))^{\mathfrak{y}}+m L_{Z} \frac{R}{2} .
\end{aligned}
$$

Using the same technique, we get

$$
\left\|\Xi_{2}(\vartheta, \mathfrak{u})\right\|_{\mathcal{P C}()} \leq m\left|w_{2}\right|+(2 m+1)\left[\frac{\left(\omega_{f} \omega_{g}+\omega_{g}^{\prime}\right) R}{2 \Gamma(\mathfrak{y}+1)\left(1-\omega_{f}^{\prime} \omega_{g}\right)}\right](\varphi(T)-\varphi(0))^{\mathfrak{y}}+m L_{Z} \frac{R}{2}
$$

Thus

$$
\begin{aligned}
\|\Xi(\mathfrak{u}, \vartheta)\|_{\mathcal{B}} & \leq\left\|\Xi_{1}(\mathfrak{u}, \vartheta)\right\|_{\mathcal{P C}())}+\left\|\Xi_{2}(\vartheta, \mathfrak{u})\right\|_{\mathcal{P C}())} \\
& \leq m\left[\left|w_{1}\right|+\left|w_{2}\right|\right]+Q_{1} R \leq R .
\end{aligned}
$$

Hence $\Xi\left(\mathbb{B}_{R}\right) \subset \mathbb{B}_{R}$.

Step 2: $\Xi$ is continuous and compact. 
Let $\left(\mathfrak{u}_{n}, \vartheta_{n}\right)$ be a sequence such that $\left(\mathfrak{u}_{n}, \vartheta_{n}\right) \rightarrow(\mathfrak{u}, \vartheta)$ in $\mathbb{B}_{R}$. Then we have

$$
\begin{aligned}
& \left|\Xi_{1}\left(\mathfrak{u}_{n}, \vartheta_{n}\right)(\sigma)-\Xi_{1}(\mathfrak{u}, \vartheta)(\sigma)\right| \\
& \leq \sum_{0<\sigma_{k}<\sigma} \mathcal{I}_{\sigma_{k-1}^{+}, \varphi}^{\mathfrak{y},}\left|\left[f\left(s, \mathfrak{u}_{n}(s), \mathcal{D}_{[\sigma]}^{\mathfrak{y}, \mathfrak{p}, \varphi} \vartheta_{n}(s)\right)-f\left(s, \mathfrak{u}(s), \mathcal{D}_{[\sigma]}^{\mathfrak{y}, \mathfrak{p}, \varphi} \vartheta(s)\right)\right]\right|(T) \\
& +\sum_{0<\sigma_{k}<\sigma} \mathcal{I}_{\sigma_{k-1}^{+}}^{\mathfrak{y}, \varphi}\left|\left[f\left(s, \mathfrak{u}_{n}(s), \mathcal{D}_{[\sigma]}^{\mathfrak{y}, \mathfrak{p}, \varphi} \vartheta_{n}(s)\right)-f\left(s, \mathfrak{u}(s), \mathcal{D}_{[\sigma]}^{\mathfrak{y}, \mathfrak{p}, \varphi} \vartheta(s)\right)\right]\right|\left(\sigma_{k}\right) \\
& +\mathcal{I}_{\sigma_{k}^{+}}^{\mathfrak{y}, \varphi}\left|\left[f\left(s, \mathfrak{u}_{n}(s), \mathcal{D}_{[\sigma]}^{\mathfrak{y}, \mathfrak{p}, \varphi} \vartheta_{n}(s)\right)-f\left(s, \mathfrak{u}(s), \mathcal{D}_{[\sigma]}^{\mathfrak{y}, \mathfrak{p}, \varphi} \vartheta(s)\right)\right]\right|(\sigma) \\
& +\sum_{0<\sigma_{k}<\sigma}\left|Z_{k} \mathfrak{u}_{n}\left(\sigma_{k}^{-}\right)-Z_{k} \mathfrak{u}\left(\sigma_{k}^{-}\right)\right| \\
& \leq m \mathcal{I}_{\sigma_{k-1}^{+}}^{\mathfrak{y}, \varphi}\left|\left[f\left(s, \mathfrak{u}_{n}(s), \mathcal{D}_{[\sigma]}^{\mathfrak{y}, \mathfrak{p}, \varphi} \vartheta_{n}(s)\right)-f\left(s, \mathfrak{u}(s), \mathcal{D}_{[\sigma]}^{\mathfrak{y}, \mathfrak{p}, \varphi} \vartheta(s)\right)\right]\right|(T) \\
& +m \mathcal{I}_{\sigma_{k-1}^{+}}^{\mathfrak{y}, \varphi}\left|\left[f\left(s, \mathfrak{u}_{n}(s), \mathcal{D}_{[\sigma]}^{\mathfrak{y}, \mathfrak{p}, \varphi} \vartheta_{n}(s)\right)-f\left(s, \mathfrak{u}(s), \mathcal{D}_{[\sigma]}^{\mathfrak{y}, \mathfrak{p}, \varphi} \vartheta(s)\right)\right]\right|\left(\sigma_{k}\right) \\
& +\mathcal{I}_{\sigma_{k}^{+}}^{\mathfrak{y}, \varphi}\left|\left[f\left(s, \mathfrak{u}_{n}(s), \mathcal{D}_{[\sigma]}^{\mathfrak{y}, \mathfrak{p}, \varphi} \vartheta_{n}(s)\right)-f\left(s, \mathfrak{u}(s), \mathcal{D}_{[\sigma]}^{\mathfrak{y}, \mathfrak{p}, \varphi} \vartheta(s)\right)\right]\right|(\sigma) \\
& +m L_{Z}\left|\left[\mathfrak{u}_{n}(\sigma)-\mathfrak{u}(\sigma)\right]\right| \\
& \leq(2 m+1) \frac{\varrho_{f}\left\|\mathfrak{u}_{n}-\mathfrak{u}\right\|_{\mathcal{P C}(J)}+\varrho_{f}^{\prime} \varrho_{g}^{\prime}\left\|\vartheta_{n}-\vartheta\right\|_{\mathcal{P C}(J)}}{\Gamma(\mathfrak{y}+1)\left(1-\varrho_{f}^{\prime} \varrho_{g}\right)}(\varphi(T)-\varphi(0))^{\mathfrak{y}} \\
& +m L_{Z}\left\|\mathfrak{u}_{n}-\mathfrak{u}\right\|_{\mathcal{P C}(J)}
\end{aligned}
$$

By the same technique we get

$$
\begin{aligned}
& \left|\Xi_{2}\left(\mathfrak{u}_{n}, \vartheta_{n}\right)(\sigma)-\Xi_{2}(\mathfrak{u}, \vartheta)(\sigma)\right| \\
& \leq(2 m+1) \frac{\varrho_{g} \varrho_{f}\left\|\mathfrak{u}_{n}-\mathfrak{u}\right\|_{\mathcal{P C}(J)}+\varrho_{g}^{\prime}\left\|\vartheta_{n}-\vartheta\right\|_{\mathcal{P C}(J)}}{\Gamma(\mathfrak{y}+1)\left(1-\varrho_{f}^{\prime} \varrho_{g}\right)}(\varphi(T)-\varphi(0))^{\mathfrak{y}} \\
& \quad+m L_{Z}\left\|\vartheta_{n}-\vartheta\right\|_{\mathcal{P C}(J)} .
\end{aligned}
$$

Thus

$$
\begin{aligned}
\| & \Xi\left(\mathfrak{u}_{n}, \vartheta_{n}\right)-\Xi(\mathfrak{u}, \vartheta) \|_{\mathcal{B}} \\
\leq & \left\|\Xi_{1}\left(\mathfrak{u}_{n}, \vartheta_{n}\right)-\Xi_{1}(\mathfrak{u}, \vartheta)\right\|_{\mathcal{P C}(J)}+\left\|\Xi_{2}\left(\mathfrak{u}_{n}, \vartheta_{n}\right)-\Xi_{2}(\mathfrak{u}, \vartheta)\right\|_{\mathcal{P C}(J)} \\
\leq & (2 m+1)\left[\frac{\left(\varrho_{f}+\varrho_{g} \varrho_{f}\right)\left\|\mathfrak{u}_{n}-\mathfrak{u}\right\|_{\mathcal{P C}(J)}+\left(\varrho_{f}^{\prime} \varrho_{g}^{\prime}+\varrho_{g}^{\prime}\right)\left\|\vartheta_{n}-\vartheta\right\|_{\mathcal{P C}(J)}}{\Gamma(\mathfrak{y}+1)\left(1-\varrho_{f}^{\prime} \varrho_{g}\right)}\right](\varphi(T)-\varphi(0))^{\mathfrak{y}} \\
& +m L_{Z}\left[\left\|\mathfrak{u}_{n}-\mathfrak{u}\right\|_{\mathcal{P C}(J)}+\left\|\vartheta_{n}-\vartheta\right\|_{\mathcal{P C}(J)}\right] \\
& \rightarrow 0 \quad \text { as }\left(\mathfrak{u}_{n}, \vartheta_{n}\right) \rightarrow(\mathfrak{u}, \vartheta) .
\end{aligned}
$$

Hence $\Xi$ is continuous. Also, the operator $\Xi$ is bounded on $\mathbb{B}_{R}$. Thus $\Xi$ is uniformly bounded on $\mathbb{B}_{R}$. Next, we prove that $\Xi$ is equicontinuous. Let $\sigma_{1}, \sigma_{2} \in J$ be such that $\sigma_{1}<$ $\sigma_{2}$. In view of $\left(\mathrm{H}_{2}\right)$, fixing $\sup _{(\sigma,(\mathfrak{u}, \vartheta)) \in J \times \mathbb{B}_{R}}|f(\sigma, \mathfrak{u}, \vartheta)|=\widehat{f}$ and $\sup _{(\sigma,(\mathfrak{u}, \vartheta)) \in J \times \mathbb{B}_{R}}|g(\sigma, \mathfrak{u}, \vartheta)|=\widehat{g}$, 
we have

$$
\begin{aligned}
\left|\Xi_{1}\left(\mathfrak{u}\left(\sigma_{2}\right), \vartheta\left(\sigma_{2}\right)\right)-\Xi_{1}\left(\mathfrak{u}\left(\sigma_{1}\right), \vartheta\left(\sigma_{1}\right)\right)\right| \\
=\mid \mathcal{I}_{\sigma_{k}^{\mathfrak{y}}, \varphi}^{\mathfrak{y}} f\left(s, \mathfrak{u}(s), \mathcal{D}_{[\sigma]}^{\mathfrak{y}, \mathfrak{p}, \varphi} \vartheta(s)\right)\left(\sigma_{2}\right) \\
\quad-\mathcal{I}_{\sigma_{k}^{+}, \varphi}^{\mathfrak{y}, \varphi}\left(s, \mathfrak{u}(s), \mathcal{D}_{[\sigma]}^{\mathfrak{y}, \mathfrak{p}, \varphi} \vartheta(s)\right)\left(\sigma_{1}\right) \mid \\
\leq \widehat{f}\left[\frac{\left(\varphi\left(\sigma_{2}\right)-\varphi\left(\sigma_{1}\right)\right)^{\mathfrak{y}}}{\Gamma(\mathfrak{y})}-\frac{\left(\varphi\left(\sigma_{1}\right)-\varphi\left(\sigma_{k}\right)\right)^{\mathfrak{y}}}{\Gamma(\mathfrak{y})}\right] .
\end{aligned}
$$

From (3.1) we have

$$
\left\|\Xi_{1}\left(\mathfrak{u}\left(\sigma_{2}\right), \vartheta\left(\sigma_{2}\right)\right)-\Xi_{1}\left(\mathfrak{u}\left(\sigma_{1}\right), \vartheta\left(\sigma_{1}\right)\right)\right\|_{\mathcal{P C}()} \rightarrow 0 \quad \text { as } \sigma_{2} \rightarrow \sigma_{1}
$$

By the same technique we get

$$
\left\|\Xi_{2}\left(\mathfrak{u}\left(\sigma_{2}\right), \vartheta\left(\sigma_{2}\right)\right)-\Xi_{2}\left(\mathfrak{u}\left(\sigma_{1}\right), \vartheta\left(\sigma_{1}\right)\right)\right\|_{\mathcal{P C}(J)} \rightarrow 0 \quad \text { as } \sigma_{2} \rightarrow \sigma_{1} .
$$

It follows from (3.2) and (3.3a) that

$$
\left\|\Xi\left(\mathfrak{u}\left(\sigma_{2}\right), \vartheta\left(\sigma_{2}\right)\right)-\Xi\left(\mathfrak{u}\left(\sigma_{1}\right), \vartheta\left(\sigma_{1}\right)\right)\right\|_{\mathcal{B}} \rightarrow 0 \quad \text { as } \sigma_{2} \rightarrow \sigma_{1} .
$$

Hence $\Xi$ is equicontinuous. By the Arzelà-Ascoli theorem we infer that $\Xi$ is compact in $\mathbb{B}_{R}$. Therefore from the above steps we conclude that $\Xi$ is completely continuous.

Step 3: The set $\digamma=\{(\mathfrak{u}, \vartheta) \in \mathcal{B}:(\mathfrak{u}, \vartheta)=\xi \Xi(\mathfrak{u}, \vartheta), \xi \in(0,1)\}$ is bounded.

Let $(\mathfrak{u}, \vartheta) \in \digamma$. Then $(\mathfrak{u}, \vartheta)=\xi \Xi(\mathfrak{u}, \vartheta)$. Now, for $\sigma \in J$, we have $\mathfrak{u}(\sigma)=\xi \Xi_{1}(\mathfrak{u}, \vartheta)$ and $\vartheta(\sigma)=\xi \Xi(\mathfrak{u}, \vartheta)$. According to our hypotheses, we attain

$$
\begin{aligned}
\left|\mathfrak{u}(\sigma)(\varphi(\sigma)-\varphi(0))^{1-\gamma}\right| & =\left|\xi \Xi_{1}(\mathfrak{u}, \vartheta)\right| \\
& \leq\left\|\Xi_{1}(\mathfrak{u}, \vartheta)\right\|_{\mathcal{P C}(J)} .
\end{aligned}
$$

By step 1 we have

$$
\begin{aligned}
\|\mathfrak{u}\|_{\mathcal{P C}())} & =\left\|\xi \Xi_{1}(\mathfrak{u}, \vartheta)\right\|_{\mathcal{P C}())} \\
& \leq\left\|\Xi_{1}(\mathfrak{u}, \vartheta)\right\|_{\mathcal{P C}(J)} \\
& \leq m\left|w_{1}\right|+(2 m+1)\left[\frac{\left(\omega_{f}+\omega_{f}^{\prime} \omega_{g}^{\prime}\right) R}{2 \Gamma(\mathfrak{y}+1)\left(1-\omega_{f}^{\prime} \omega_{g}\right)}\right](\varphi(T)-\varphi(0))^{\mathfrak{y}}+m L_{Z} \frac{R}{2}
\end{aligned}
$$

and

$$
\|\vartheta\|_{\mathcal{P C}(J)} \leq m\left|w_{2}\right|+(2 m+1)\left[\frac{\left(\omega_{f} \omega_{g}+\omega_{g}^{\prime}\right) R}{2 \Gamma(\mathfrak{y}+1)\left(1-\omega_{f}^{\prime} \omega_{g}\right)}\right](\varphi(T)-\varphi(0))^{\mathfrak{y}}+m L_{Z} \frac{R}{2}
$$

From (3.4) and (3.5) we have

$$
\|(\mathfrak{u}, \vartheta)\|_{\mathcal{B}}=\|\mathfrak{u}\|_{\mathcal{P C}())}+\|\vartheta\|_{\mathcal{P C}())} \leq R .
$$


Hence the set $\digamma$ is bounded. According to the above steps, together with Theorem 2.7, we conclude that $\Xi$ has at least one fixed point. Consequently, system (1.1) has at least one solution on $J$.

In the following theorem, we prove the uniqueness of solutions to system (1.1) by using Theorem 2.8.

Theorem 3.2 Assume that $\left(H_{1}\right)-\left(H_{3}\right)$ hold. If

$$
\mathcal{Q}=(2 m+1) \rho+m L_{Z}<1
$$

where $\rho=\max \left\{\rho_{1}, \rho_{2}\right\}$ with

$$
\begin{aligned}
& \rho_{1}=\frac{\varrho_{f}\left(1+\varrho_{g}\right)}{\Gamma(\mathfrak{y}+1)\left(1-\varrho_{f}^{\prime} \varrho_{g}\right)}, \\
& \rho_{2}=\frac{\varrho_{g}^{\prime}\left(1+\varrho_{f}^{\prime}\right)}{\Gamma(\mathfrak{y}+1)\left(1-\varrho_{f}^{\prime} \varrho_{g}\right)},
\end{aligned}
$$

then system (1.1) has a unique solution.

Proof Consider the closed ball $\mathbb{B}_{R}$ defined in Theorem 3.1. First, we show that $\Xi\left(\mathbb{B}_{R}\right) \subset \mathbb{B}_{R}$. By the first step in Theorem 3.1 we have $\Xi\left(\mathbb{B}_{R}\right) \subset \mathbb{B}_{R}$. Next, we need to prove that $\Xi$ is a contraction map. Indeed, for $(\mathfrak{u}, \vartheta),(\widehat{\mathfrak{u}}, \widehat{\vartheta}) \in \mathbb{B}_{R}$ and $\sigma \in J$, we obtain

$$
\begin{aligned}
& \left|\left(\Xi_{1}(\mathfrak{u}, \vartheta)(\sigma)-\Xi_{1}(\widehat{\mathfrak{u}}, \widehat{\vartheta})(\sigma)\right)\right| \\
& \leq \sum_{0<\sigma_{k}<\sigma} \frac{\left(\varphi\left(\sigma_{k}\right)-\varphi\left(\sigma_{k-1}\right)\right)^{1-\gamma}}{\left(\varphi(T)-\varphi\left(\sigma_{k-1}\right)\right)^{1-\gamma}} \\
& \times\left[\mathcal{I}_{\sigma_{k-1}^{+}, \varphi}^{\mathfrak{y}, \varphi}\left|f\left(s, \mathfrak{u}(s), \mathcal{D}_{[\sigma]}^{\mathfrak{y}, \mathfrak{p}, \varphi} \vartheta(s)\right)-f\left(s, \widehat{\mathfrak{u}}(s), \mathcal{D}_{[\sigma]}^{\mathfrak{y}, \mathfrak{p}, \varphi} \widehat{\vartheta}(s)\right)\right|(T)\right] \\
& +\sum_{0<\sigma_{k}<\sigma} \mathcal{I}_{\sigma_{k-1}^{+}, \varphi}^{\mathfrak{y},}\left|f\left(s, \mathfrak{u}(s), \mathcal{D}_{[\sigma]}^{\mathfrak{y}, \mathfrak{p}, \varphi} \vartheta(s)\right)-f\left(s, \widehat{\mathfrak{u}}(s), \mathcal{D}_{[\sigma]}^{\mathfrak{y}, \mathfrak{p}, \varphi} \widehat{\vartheta}(s)\right)\right|\left(\sigma_{k}\right) \\
& +\mathcal{I}_{\sigma_{k}^{+}}^{\mathfrak{y}, \varphi}\left|f\left(s, \mathfrak{u}(s), \mathcal{D}_{[\sigma]}^{\mathfrak{y}, \mathfrak{p}, \varphi} \vartheta(s)\right)-f\left(s, \widehat{\mathfrak{u}}(s), \mathcal{D}_{[\sigma]}^{\mathfrak{y}, \mathfrak{p}, \varphi} \widehat{\vartheta}(s)\right)\right|(\sigma) \\
& +\sum_{0<\sigma_{k}<\sigma}\left|Z_{k} \mathfrak{u}\left(\sigma_{k}^{-}\right)-Z_{k} \widehat{\mathfrak{u}}\left(\sigma_{k}^{-}\right)\right| \\
& \leq m \mathcal{I}_{\sigma_{k-1}^{+}, \varphi}^{\mathfrak{y},}\left|\left[f\left(s, \mathfrak{u}(s), \mathcal{D}_{[\sigma]}^{\mathfrak{y}, \mathfrak{p}, \varphi} \vartheta(s)\right)-f\left(s, \widehat{\mathfrak{u}}(s), \mathcal{D}_{[\sigma]}^{\mathfrak{y}, \mathfrak{p}, \varphi} \widehat{\vartheta}(s)\right)\right]\right|(T) \\
& +m \mathcal{I}_{\sigma_{k-1}^{+}, \varphi}^{\mathfrak{y}, \varphi}\left|\left[f\left(s, \mathfrak{u}(s), \mathcal{D}_{[\sigma]}^{\mathfrak{y}, \mathfrak{p}, \varphi} \vartheta(s)\right)-f\left(s, \widehat{\mathfrak{u}}(s), \mathcal{D}_{[\sigma]}^{\mathfrak{y}, \mathfrak{p}, \varphi} \widehat{\vartheta}(s)\right)\right]\right|\left(\sigma_{k}\right) \\
& +\mathcal{I}_{\sigma_{k}^{+}}^{\mathfrak{y}, \varphi}\left|\left[f\left(s, \mathfrak{u}(s), \mathcal{D}_{[\sigma]}^{\mathfrak{y}, \mathfrak{p}, \varphi} \vartheta(s)\right)-f\left(s, \widehat{\mathfrak{u}}(s), \mathcal{D}_{[\sigma]}^{\mathfrak{y}, \mathfrak{p}, \varphi} \widehat{\vartheta}(s)\right)\right]\right|(\sigma) \\
& +m\left|Z_{k} \mathfrak{u}\left(\sigma_{k}^{-}\right)-Z_{k} \widehat{\mathfrak{u}}\left(\sigma_{k}^{-}\right)\right| \\
& \leq(2 m+1)\left[\frac{\left(\varrho_{f}\|\mathfrak{u}-\widehat{\mathfrak{u}}\|_{\mathcal{P C}()}+\varrho_{f}^{\prime} \varrho_{g}^{\prime}\|\vartheta-\widehat{\vartheta}\|_{\mathcal{P C}())}\right.}{\Gamma(\mathfrak{y}+1)\left(1-\varrho_{f}^{\prime} \varrho_{g}\right)}\right](\varphi(T)-\varphi(0))^{\mathfrak{y}} \\
& +m L_{Z}\|\mathfrak{u}-\widehat{\mathfrak{u}}\|_{\mathcal{P C}()},
\end{aligned}
$$


and, consequently, we obtain

$$
\begin{aligned}
& \left\|\Xi_{1}(\mathfrak{u}, \vartheta)(\sigma)-\Xi_{1}(\widehat{\mathfrak{u}}, \widehat{\vartheta})\right\|_{\mathcal{P C}())} \\
& \leq(2 m+1)\left[\frac{\varrho_{f}\|\mathfrak{u}-\widehat{\mathfrak{u}}\|_{\mathcal{P C}())}+\varrho_{f}^{\prime} \varrho_{g}^{\prime}\|\vartheta-\widehat{\vartheta}\|_{\mathcal{P C}())}}{\Gamma(\mathfrak{y}+1)\left(1-\varrho_{f}^{\prime} \varrho_{g}\right)}\right](\varphi(T)-\varphi(0))^{\mathfrak{y}} \\
& \quad+m L_{Z}\|\mathfrak{u}-\widehat{\mathfrak{u}}\|_{\mathcal{P C}(J)} .
\end{aligned}
$$

By the same way we obtain

$$
\begin{aligned}
& \left\|\Xi_{2}(\mathfrak{u}, \vartheta)(\sigma)-\Xi_{2}(\widehat{\mathfrak{u}}, \widehat{\vartheta})\right\|_{\mathcal{P C}())} \\
& \leq(2 m+1)\left[\frac{\varrho_{f} \varrho_{g}\|\mathfrak{u}-\widehat{\mathfrak{u}}\|_{\mathcal{P C}()}+\varrho_{g}^{\prime}\|\vartheta-\widehat{\vartheta}\|_{\mathcal{P C}())}}{\Gamma(\mathfrak{y}+1)\left(1-\varrho_{f}^{\prime} \varrho_{g}\right)}\right](\varphi(T)-\varphi(0))^{\mathfrak{y}} \\
& \quad+m L_{Z}\|\vartheta-\widehat{\vartheta}\|_{\mathcal{P C}())}
\end{aligned}
$$

From (3.7) and (3.8) it follows that

$$
\begin{aligned}
& \|\Xi(\mathfrak{u}, \vartheta)(\sigma)-\Xi(\widehat{\mathfrak{u}}, \widehat{\vartheta})\|_{\mathcal{B}} \\
& \leq\left\|\Xi_{1}(\mathfrak{u}, \vartheta)(\sigma)-\Xi_{1}(\widehat{\mathfrak{u}}, \widehat{\vartheta})\right\|_{\mathcal{P C}())}+\left\|\Xi_{2}(\mathfrak{u}, \vartheta)(\sigma)-\Xi_{2}(\widehat{\mathfrak{u}}, \widehat{\vartheta})\right\|_{\mathcal{P C}(J)} \\
& \leq(2 m+1)\left[\frac{\varrho_{f}\left(1+\varrho_{g}\right)\|\mathfrak{u}-\widehat{\mathfrak{u}}\|_{\mathcal{P C}())}}{\Gamma(\mathfrak{y}+1)\left(1-\varrho_{f}^{\prime} \varrho_{g}\right)}+\frac{\varrho_{g}^{\prime}\left(1+\varrho_{f}^{\prime}\right)\|\vartheta-\widehat{\vartheta}\|_{\mathcal{P C}())}}{\Gamma(\mathfrak{y}+1)\left(1-\varrho_{f}^{\prime} \varrho_{g}\right)}\right](\varphi(T)-\varphi(0))^{\mathfrak{y}} \\
& +m L_{Z}\left[\|\mathfrak{u}-\widehat{\mathfrak{u}}\|_{\mathcal{P C}())}+\|\vartheta-\widehat{\vartheta}\|_{\mathcal{P C}())}\right] \\
& \leq(2 m+1)\left[\rho_{1}\|\mathfrak{u}-\widehat{\mathfrak{u}}\|_{\mathcal{P C}())}+\rho_{2}\|\vartheta-\widehat{\vartheta}\|_{\mathcal{P C}())}\right](\varphi(T)-\varphi(0))^{\mathfrak{y}} \\
& +m L_{Z}\left[\|\mathfrak{u}-\widehat{\mathfrak{u}}\|_{\mathcal{P C}())}+\|\vartheta-\widehat{\vartheta}\|_{\mathcal{P C}())}\right] \\
& \leq\left[(2 m+1) \rho+m L_{Z}\right]\left[\|\mathfrak{u}-\widehat{\mathfrak{u}}\|_{\mathcal{P C}(J)}+\|\vartheta-\widehat{\vartheta}\|_{\mathcal{P C}()}\right] \\
& \leq \mathcal{Q}\|(\mathfrak{u}, \vartheta)-(\widehat{u}, \widehat{\vartheta})\|_{\mathcal{B}}
\end{aligned}
$$

Thus the operator $\Xi$ is a contraction. So by Theorem 2.8 system (1.1) has a unique solution.

\section{Stability analysis}

To state the main theorem, we need the following definitions. Let $\epsilon_{i}>0$ and $\lambda_{\phi_{i}}: J \rightarrow[0, \infty)$ $(i=1,2)$ be continuous functions. We consider the following inequalities:

$$
\begin{aligned}
& \left|\mathcal{D}_{[\sigma]}^{\mathfrak{y}, \mathfrak{p}, \varphi} \widehat{\mathfrak{u}}(\sigma)-f\left(\sigma, \widehat{\mathfrak{u}}(\sigma), \mathcal{D}_{[\sigma]}^{\mathfrak{y}, \mathfrak{p}, \varphi} \widehat{\vartheta}(\sigma)\right)\right| \leq \epsilon_{1}, \\
& \left|\mathcal{D}_{[\sigma]}^{\mathfrak{y}, \mathfrak{p}, \varphi} \widehat{\vartheta}(\sigma)-f\left(\sigma, \mathcal{D}_{0}^{\mathfrak{y}, \mathfrak{p}, \varphi} \widehat{\mathfrak{u}}(\sigma), \widehat{\vartheta}(\sigma)\right)\right| \leq \epsilon_{2}, \\
& \left|\mathcal{D}_{[\sigma]}^{\mathfrak{y}, \mathfrak{p}, \varphi} \widehat{\mathfrak{u}}(\sigma)-f\left(\sigma, \widehat{\mathfrak{u}}(\sigma), \mathcal{D}_{[\sigma]}^{\mathfrak{y}, \mathfrak{p}, \varphi} \widehat{\vartheta}(\sigma)\right)\right| \leq \epsilon_{1} \lambda_{\phi_{1}}(\sigma), \\
& \left|\mathcal{D}_{[\sigma]}^{\mathfrak{y}, \mathfrak{p}, \varphi} \widehat{\vartheta}(\sigma)-f\left(\sigma, \mathcal{D}_{0}^{\mathfrak{y}, \mathfrak{p}, \varphi} \widehat{\mathfrak{u}}(\sigma), \widehat{\vartheta}(\sigma)\right)\right| \leq \epsilon_{2} \lambda_{\phi_{2}}(\sigma) .
\end{aligned}
$$

Definition 4.1 ([39]) System (1.1) is UH stable if there exists a real number $\mathcal{M}>0$ such that for each $\epsilon=\max \left\{\epsilon_{1}, \epsilon_{2}\right\}>0$, there exists a solution $(\widehat{\mathfrak{u}}, \widehat{\vartheta}) \in \mathcal{B}$ of inequalities (4.1) and 
(4.2) corresponding to a solution $(\mathfrak{u}, \vartheta) \in \mathcal{B}$ of system (1.1) such that

$$
\|(\widehat{\mathfrak{u}}, \widehat{\vartheta})-(\mathfrak{u}, \vartheta)\|_{\mathcal{B}} \leq \mathcal{M} \epsilon, \quad \varkappa \in J
$$

Definition 4.2 ([39]) System (1.1) is UHR stable with respect to the nondecreasing function $\lambda_{\phi}(\sigma)=\max _{\varkappa \in J}\left\{\lambda_{\phi_{1}}(\sigma), \lambda_{\phi 2}(\sigma)\right\}$ if there exists a real number $\mathcal{N}>0$ such that for each solution $(\widehat{\mathfrak{u}}, \widehat{\vartheta}) \in \mathcal{B}$ of inequalities (4.3) and (4.4), there exists a solution $(\mathfrak{u}, \vartheta) \in \mathcal{B}$ of system (1.1) such that

$$
\|(\widehat{\mathfrak{u}}, \widehat{\vartheta})-(\mathfrak{u}, \vartheta)\|_{\mathcal{B}} \leq \mathcal{N} \in \lambda_{\phi}(\varkappa), \quad \varkappa \in J
$$

Remark 4.3 A function $(\widehat{\mathfrak{u}}, \widehat{\vartheta}) \in \mathcal{B}$ is a solution of inequalities (4.1) and (4.2) if and only if there exist functions $z_{1}, z_{2} \in \mathcal{P C}(J)$ such that

(i) $\left\{\begin{array}{l}\left|z_{1}(\varkappa)\right| \leq \epsilon_{1}, \sigma \in J, \\ \left|z_{2}(\varkappa)\right| \leq \epsilon_{2}, \sigma \in J,\end{array}\right.$

(ii) $\left\{\begin{array}{l}\mathcal{D}_{[\sigma]}^{\mathfrak{y}, \mathfrak{p}, \varphi} \widehat{\mathfrak{u}}(\sigma)=f\left(\sigma, \widehat{\mathfrak{u}}(\sigma), \mathcal{D}_{[\sigma]}^{\mathfrak{y}, \mathfrak{p}, \varphi} \widehat{\vartheta}(\sigma)\right)+z_{1}(\sigma), \sigma \in J, \\ \mathcal{D}_{[\sigma]}^{\mathfrak{y}, \mathfrak{p}, \varphi} \widehat{\vartheta}(\sigma)=g\left(\sigma, \mathcal{D}_{[\sigma]}^{\mathfrak{y}, \mathfrak{p}, \varphi} \widehat{\widehat{\mathfrak{u}}}(\sigma), \widehat{\vartheta}(\sigma)\right)+z_{2}(\sigma), \sigma \in J .\end{array}\right.$

Lemma 4.4 Let $\mathfrak{y} \in(0,1)$ and $\mathfrak{p} \in[0,1]$. If a function $(\widehat{\mathfrak{u}}, \widehat{\vartheta}) \in \mathcal{B}$ satisfies inequalities (4.1) and (4.2), then $(\widehat{\mathfrak{u}}, \widehat{\vartheta})$ satisfies the following integral inequalities:

$$
\left\{\begin{array}{l}
\left|\widehat{\mathfrak{u}}(\sigma)-\mathcal{A}_{\widehat{\mathfrak{u}}}-\mathcal{I}_{\sigma_{k}^{+}}^{\mathfrak{y}, \varphi} f\left(s, \widehat{\mathfrak{u}}(s), \mathcal{D}_{[\sigma]}^{\mathfrak{y}, \mathfrak{p}, \varphi} \widehat{\vartheta}(s)\right)(\sigma)\right| \leq \epsilon_{1} K, \\
\left|\widehat{\vartheta}(\sigma)-\mathcal{A}_{\widehat{\vartheta}}-\mathcal{I}_{\sigma_{k}^{+}, \varphi}^{\mathfrak{y}, \varphi} g\left(s, \mathcal{D}_{[\sigma]}^{\mathfrak{y}, \mathfrak{p}, \varphi} \widehat{\mathfrak{u}}(s), \widehat{\vartheta}(s),\right)(\sigma)\right| \leq \epsilon_{2} K,
\end{array}\right.
$$

where

$$
\left\{\begin{aligned}
\mathcal{A}_{\widehat{\mathfrak{u}}}:= & \sum_{0<\sigma_{k}<\sigma} \frac{\left(\varphi\left(\sigma_{k}\right)-\varphi\left(\sigma_{k-1}\right)\right)^{\gamma-1}}{\left(\varphi(T)-\varphi\left(\sigma_{k-1}\right)\right)^{\gamma-1}}\left[w_{1}-\mathcal{I}_{\sigma_{k-1}^{\mathfrak{y}, \varphi}}^{\mathfrak{y}} f\left(s, \widehat{\mathfrak{u}}(s), \mathcal{D}_{[\sigma]}^{\mathfrak{y}, \mathfrak{p}, \varphi} \widehat{\vartheta}(s)\right)(T)\right] \\
& -\sum_{0<\sigma_{k}<\sigma} \mathcal{I}_{\sigma_{k-1}^{+}, \varphi} f\left(s, \widehat{\mathfrak{u}}(s), \mathcal{D}_{[\sigma]}^{\mathfrak{y}, \mathfrak{p}, \varphi} \widehat{\vartheta}(s)\right)\left(\sigma_{k}\right)-\sum_{0<\sigma_{k}<\sigma} Z_{k} \widehat{\mathfrak{u}}\left(\sigma_{k}^{-}\right), \\
\mathcal{A}_{\widehat{\vartheta}}:= & \sum_{0<\sigma_{k}<\sigma} \frac{\left(\varphi\left(\sigma_{k}\right)-\varphi\left(\sigma_{k-1}\right)\right)^{\gamma-1}}{\left(\varphi(T)-\varphi\left(\sigma_{k-1}\right)\right)^{\gamma-1}}\left[w_{2}-\mathcal{I}_{\sigma_{k-1}^{\mathfrak{y}}, \varphi}^{\mathfrak{y}, \varphi} g\left(s, \mathcal{D}_{[\sigma]}^{\mathfrak{y}, \mathfrak{p}, \varphi} \widehat{\mathfrak{u}}(s), \widehat{\vartheta}(s)\right)(T)\right] \\
& -\sum_{0<\sigma_{k}<\sigma} \mathcal{I}_{\sigma_{k-1}^{\mathfrak{y}, \varphi}}^{+} g\left(s, \mathcal{D}_{[\sigma]}^{\mathfrak{y}, \mathfrak{p}, \varphi} \widehat{\mathfrak{u}}(s), \widehat{\vartheta}(s)\right)\left(\sigma_{k}\right)-\sum_{0<\sigma_{k}<\sigma} Z_{k} \widehat{\vartheta}\left(\sigma_{k}^{-}\right),
\end{aligned}\right.
$$

and

$$
K:=(2 m+1) \frac{(\varphi(T)-\varphi(0))^{\mathfrak{y}}}{\Gamma(\mathfrak{y}+1)} .
$$

Proof Indeed, by Remark 4.3 we have

$$
\begin{cases}\mathcal{D}_{[\sigma]}^{\mathfrak{y}, \mathfrak{p}, \varphi} \widehat{\mathfrak{u}}(\sigma)=f\left(\sigma, \widehat{\mathfrak{u}}(\sigma), \mathcal{D}_{[\sigma]}^{\mathfrak{y}, \mathfrak{p}, \varphi} \widehat{\vartheta}(\sigma)\right)+z_{1}(\sigma), & \sigma \in J, \\ \mathcal{D}_{[\sigma]}^{\mathfrak{y}, \mathfrak{p}, \varphi} \widehat{\vartheta}(\sigma)=g\left(\sigma, \mathcal{D}_{[\sigma]}^{\mathfrak{y}, \mathfrak{p}, \varphi} \widehat{\mathfrak{u}}(\sigma), \widehat{\vartheta}(\sigma)\right)+z_{2}(\sigma), & \sigma \in J .\end{cases}
$$


Then, for $\sigma \in\left(\sigma_{k}, \sigma_{k+1}\right], k=1, \ldots, m$, we get

$$
\left\{\begin{array}{c}
\left|\widehat{\mathfrak{u}}(\sigma)-\mathcal{A}_{\widehat{\mathfrak{u}}}-\mathcal{I}_{\sigma_{k}^{+}}^{\mathfrak{y}, \varphi} f\left(s, \widehat{\mathfrak{u}}(s), \mathcal{D}_{0}^{\mathfrak{y}, \mathfrak{p}, \varphi} \widehat{\vartheta}(s)\right)(\sigma)\right| \\
\leq \sum_{0<\sigma_{k}<\sigma} \frac{\left(\varphi\left(\sigma_{k}\right)-\varphi\left(\sigma_{k-1}\right)\right)^{\gamma-1}}{\left(\varphi(T)-\varphi\left(\sigma_{k-1}\right) \gamma^{\gamma-1}\right.}\left[\mathcal{I}_{\sigma_{k-1}^{\mathfrak{y}, \varphi}}^{+}\left|z_{1}(s)\right|(T)\right] \\
\quad+\sum_{0<\sigma_{k}<\sigma} \mathcal{I}_{\sigma_{k-1}^{\mathfrak{y}, \varphi}}\left|z_{1}(s)\right|\left(\sigma_{k}\right)+\mathcal{I}_{\sigma_{k}^{+}, \varphi}^{\mathfrak{y}, \varphi}\left|z_{1}(s)\right|(\sigma), \\
\widehat{\mid \vartheta}(\sigma)-\mathcal{A}_{\widehat{\vartheta}}-\mathcal{I}_{\sigma_{k}^{+}, \varphi}^{\mathfrak{y}} g\left(s, \mathcal{D}_{0}^{\mathfrak{y}, \mathfrak{p}, \varphi} \widehat{\mathfrak{u}}(s), \widehat{\vartheta}(s)\right)(\sigma) \mid \\
\leq \sum_{0<\sigma_{k}<\sigma} \frac{\left(\varphi\left(\sigma_{k}\right)-\varphi\left(\sigma_{k-1}\right)\right)^{\gamma-1}}{\left(\varphi(T)-\varphi\left(\sigma_{k-1}\right) \gamma^{\gamma-1}\right.}\left[\mathcal{I}_{\sigma_{k-1}^{\mathfrak{y}, \varphi}}\left|z_{2}(s)\right|(T)\right] \\
\quad+\sum_{0<\sigma_{k}<\sigma} \mathcal{I}_{\sigma_{k-1}^{+}, \varphi}^{\mathfrak{y},}\left|z_{2}(s)\right|\left(\sigma_{k}\right)+\mathcal{I}_{\sigma_{k}^{+}, \varphi}^{\mathfrak{y}, \varphi}\left|z_{2}(s)\right|(\sigma) .
\end{array}\right.
$$

It follows that

$$
\left\{\begin{array}{l}
\left|\widehat{\mathfrak{u}}(\sigma)-\mathcal{A}_{\widehat{\mathfrak{u}}}-\mathcal{I}_{\sigma_{k}^{+}}^{\mathfrak{y}, \varphi} f\left(s, \widehat{\mathfrak{u}}(s), \mathcal{D}_{[\sigma]}^{\mathfrak{y}, \mathfrak{p}, \varphi} \widehat{\vartheta}(s)\right)(\sigma)\right| \leq \epsilon_{1} K, \\
\left|\widehat{\vartheta}(\sigma)-\mathcal{A}_{\widehat{\vartheta}}-\mathcal{I}_{\sigma_{k}^{+}, \varphi}^{\mathfrak{y}} g\left(s, \mathcal{D}_{[\sigma]}^{\mathfrak{y}, \mathfrak{p}, \varphi} \widehat{\mathfrak{u}}(s), \widehat{\vartheta}(s),\right)(\sigma)\right| \leq \epsilon_{2} K .
\end{array}\right.
$$

In the forthcoming theorem, we prove the stability results for system (1.1).

Theorem 4.5 Assume that $\left(H_{1}\right)$ and $\left(H_{2}\right)$ hold. Then

$$
\begin{cases}\mathcal{D}_{[\sigma]}^{\mathfrak{y}, \mathfrak{p}, \varphi} \mathfrak{u}(\sigma)=f\left(\sigma, \mathfrak{u}(\sigma), \mathcal{D}_{[\sigma]}^{\mathfrak{y}, \mathfrak{p}, \varphi} \vartheta(\sigma)\right), & \sigma \in J, \\ \mathcal{D}_{[\sigma]}^{\mathfrak{y}, \mathfrak{p}, \varphi} \vartheta(\sigma)=g\left(\sigma, \mathcal{D}_{[\sigma]}^{\mathfrak{y}, \mathfrak{p}, \varphi} \mathfrak{u}(\sigma), \vartheta(\sigma)\right), & \sigma \in J\end{cases}
$$

are UH stable, provided that $\Delta=\left(1-\Lambda_{1 f}\right)\left(1-\Lambda_{2 g}\right)-\Lambda_{1 g} \Lambda_{2 f} \neq 0$, where

$$
\begin{array}{ll}
\Lambda_{1 f}=\frac{\varrho_{f}(\varphi(T)-\varphi(0))^{\mathfrak{y}}}{\Gamma(\mathfrak{y}+1)\left(1-\varrho_{f}^{\prime} \varrho_{g}\right)}, & \Lambda_{2 f}=\frac{(\varphi(T)-\varphi(0))^{\mathfrak{y}} \varrho_{f} \varrho_{g}}{\Gamma(\mathfrak{y}+1)\left(1-\varrho_{f}^{\prime} \varrho_{g}\right)}, \\
\Lambda_{1 g}=\frac{(\varphi(T)-\varphi(0))^{\mathfrak{y}} \varrho_{f}^{\prime} \varrho_{g}^{\prime}}{\Gamma(\mathfrak{y}+1)\left(1-\varrho_{f}^{\prime} \varrho_{g}\right)}, & \Lambda_{2 g}=\frac{(\varphi(T)-\varphi(0))^{\mathfrak{y}} \varrho_{g}^{\prime}}{\Gamma(\mathfrak{y}+1)\left(1-\varrho_{f}^{\prime} \varrho_{g}\right)}
\end{array}
$$

Proof Let $\epsilon=\max \left\{\epsilon_{1}, \epsilon_{2}\right\}>0$, let $(\widehat{\mathfrak{u}}, \widehat{\vartheta}) \in \mathcal{B}$ be functions satisfying inequalities (4.1) and (4.2), and let $(\mathfrak{u}, \vartheta) \in \mathcal{B}$ be the unique solution of the following system

$$
\left\{\begin{array}{l}
\mathcal{D}_{[\sigma]}^{\mathfrak{y}, \mathfrak{p}, \varphi} \mathfrak{u}(\sigma)=f\left(\sigma, \mathfrak{u}(\sigma), \mathcal{D}_{[\sigma]}^{\mathfrak{y}, \mathfrak{p}, \varphi} \vartheta(\sigma)\right), \quad \sigma \in J:=[0, T], \sigma \neq \sigma_{k}, k=1, \ldots, m, \\
\mathcal{D}_{[\sigma]}^{\mathfrak{y}, \mathfrak{p}, \varphi} \vartheta(\sigma)=g\left(\sigma, \mathcal{D}_{[\sigma]}^{\mathfrak{y}, \mathfrak{p}, \varphi} \mathfrak{u}(\sigma), \vartheta(\sigma)\right), \quad \sigma \in J:=[0, T], \sigma \neq \sigma_{k}, k=1, \ldots, m, \\
\left.\Delta \mathfrak{u}\right|_{\sigma=\sigma_{k}}=\left.\Delta \widehat{\mathfrak{u}}\right|_{\sigma=\sigma_{k}}=Z_{k} \widehat{\mathfrak{u}}\left(\sigma_{k}^{-}\right), \quad k=1, \ldots, m, \\
\left.\Delta \vartheta\right|_{\sigma=\sigma_{k}}=\left.\Delta \widehat{\vartheta}\right|_{\sigma=\sigma_{k}}=Z_{k} \widehat{\vartheta}\left(\sigma_{k}^{-}\right), \quad k=1, \ldots, m, \\
\widehat{\mathfrak{u}}(T)=\mathfrak{u}(T)=w_{1}, \quad \widehat{\vartheta}(T)=\vartheta(T)=w_{2} .
\end{array}\right.
$$

Then by Theorem 3.1 we have

$$
\left\{\begin{array}{l}
\mathfrak{u}(\sigma)=\mathcal{A}_{\mathfrak{u}}+\mathcal{I}_{\sigma_{k}^{+}}^{\mathfrak{y}, \varphi} f\left(s, \mathfrak{u}(s), \mathcal{D}_{[\sigma]}^{\mathfrak{y}, \mathfrak{p}, \varphi} \vartheta(s)\right)(\sigma), \\
\vartheta(\sigma)=\mathcal{A}_{\vartheta}+\mathcal{I}_{\sigma_{k}^{+}, \varphi}^{\mathfrak{y}, \varphi}\left(s, \mathcal{D}_{[\sigma]}^{\mathfrak{y}, \mathfrak{p}, \varphi} \mathfrak{u}(s), \vartheta(s)\right)(\sigma)
\end{array}\right.
$$


Since

$$
\left\{\begin{array}{l}
\left.\Delta \mathfrak{u}\right|_{\sigma=\sigma_{k}}=\left.\Delta \widehat{\mathfrak{u}}\right|_{\sigma=\sigma_{k}}=Z_{k} \widehat{\mathfrak{u}}\left(\sigma_{k}^{-}\right), \quad k=1, \ldots, m, \\
\left.\Delta \vartheta\right|_{\sigma=\sigma_{k}}=\left.\Delta \widehat{\vartheta}\right|_{\sigma=\sigma_{k}}=Z_{k} \widehat{\vartheta}\left(\sigma_{k}^{-}\right), \quad k=1, \ldots, m, \\
\widehat{\mathfrak{u}}(T)=\mathfrak{u}(T)=w_{1}, \\
\widehat{\vartheta}(T)=\vartheta(T)=w_{2},
\end{array}\right.
$$

we can easily prove that $\mathcal{A}_{\mathfrak{u}}=\mathcal{A}_{\widehat{\mathfrak{u}}}$ and $\mathcal{A}_{\vartheta}=\mathcal{A}_{\widehat{\vartheta}}$. Hence from $\left(\mathrm{H}_{2}\right)$ and Lemma 4.4, for each $\sigma \in J$, we have

$$
\begin{aligned}
|\widehat{\mathfrak{u}}(\sigma)-\mathfrak{u}(\sigma)|= & \mid \widehat{\mathfrak{u}}(\sigma)-\mathcal{A}_{\widehat{\mathfrak{u}}}-\mathcal{I}_{\sigma_{k}^{+}}^{\mathfrak{y}, \varphi} f\left(s, \mathfrak{u}(s), \mathcal{D}_{[\sigma]}^{\mathfrak{y}, \mathfrak{p}, \varphi} \vartheta(s)\right)(\sigma) \\
& -\mathcal{I}_{\sigma_{k}^{+}}^{\mathfrak{y}, \varphi} f\left(s, \widehat{\mathfrak{u}}(s), \mathcal{D}_{[\sigma]}^{\mathfrak{y}, \mathfrak{p}, \varphi} \widehat{\vartheta}(s)\right)(\sigma)+f\left(s, \widehat{\mathfrak{u}}(s), \mathcal{D}_{[\sigma]}^{\mathfrak{y}, \mathfrak{p}, \varphi} \widehat{\vartheta}(s)\right)(\sigma) \mid \\
\leq & \left|\widehat{\mathfrak{u}}(\sigma)-\mathcal{A}_{\widehat{\mathfrak{u}}}-\mathcal{I}_{\sigma_{k}^{+}, \varphi}^{\mathfrak{y}, \varphi} f\left(s, \widehat{\mathfrak{u}}(s), \mathcal{D}_{[\sigma]}^{\mathfrak{y}, \mathfrak{p}, \varphi} \widehat{\vartheta}(s)\right)(\sigma)\right| \\
& +\mathcal{I}_{\sigma_{k}^{+}}^{\mathfrak{y}, \varphi}\left|f\left(s, \widehat{\mathfrak{u}}(s), \mathcal{D}_{[\sigma]}^{\mathfrak{y}, \mathfrak{p}, \varphi} \widehat{\vartheta}(s)\right)(\sigma)-f\left(s, \mathfrak{u}(s), \mathcal{D}_{[\sigma]}^{\mathfrak{y}, \mathfrak{p}, \varphi} \vartheta(s)\right)(\sigma)\right| \\
\leq & K \epsilon_{1}++\mathcal{I}_{\sigma_{k}^{+}}^{\mathfrak{y}, \varphi}\left|f\left(s, \widehat{\mathfrak{u}}(s), \mathcal{D}_{[\sigma]}^{\mathfrak{y}, \mathfrak{p}, \varphi} \widehat{\vartheta}(s)\right)(\sigma)-f\left(s, \mathfrak{u}(s), \mathcal{D}_{[\sigma]}^{\mathfrak{y}, \mathfrak{p}, \varphi} \vartheta(s)\right)(\sigma)\right|
\end{aligned}
$$

and

$$
\begin{aligned}
& |\widehat{\vartheta}(\sigma)-\vartheta(\sigma)|=\mid \widehat{\vartheta}(\sigma)-\mathcal{A}_{\widehat{\vartheta}}-\mathcal{I}_{\sigma_{k}^{+}}^{\mathfrak{y}, \varphi} g\left(s, \mathcal{D}_{[\sigma]}^{\mathfrak{y}, \mathfrak{p}, \varphi} \mathfrak{u}(s), \vartheta(s)\right)(\sigma) \\
& -\mathcal{I}_{\sigma_{k}^{+}}^{\mathfrak{y}, \varphi} g\left(s, \mathcal{D}_{[\sigma]}^{\mathfrak{y}, \mathfrak{p}, \varphi} \widehat{\mathfrak{u}}(s), \widehat{\vartheta}(s)\right)(\sigma)+g\left(s, \mathcal{D}_{[\sigma]}^{\mathfrak{y}, \mathfrak{p}, \varphi} \widehat{\mathfrak{u}}(s), \widehat{\vartheta}(s)\right)(\sigma) \mid
\end{aligned}
$$

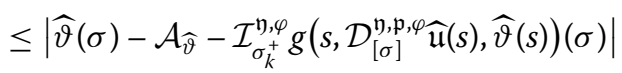

$$
\begin{aligned}
& +\mathcal{I}_{\sigma_{k}^{+}}^{\mathfrak{y}, \varphi}\left|g\left(s, \mathcal{D}_{[\sigma]}^{\mathfrak{y}, \mathfrak{p}, \varphi} \widehat{\mathfrak{u}}(s), \widehat{\vartheta}(s)\right)(\sigma)-g\left(s, \mathcal{D}_{[\sigma]}^{\mathfrak{y}, \mathfrak{p}, \varphi} \mathfrak{u}(s), \vartheta(s)\right)(\sigma)\right| \\
& \leq K \epsilon_{2}+\mathcal{I}_{\sigma_{k}^{+}}^{\mathfrak{y}, \varphi}\left|g\left(s, \mathcal{D}_{[\sigma]}^{\mathfrak{y}, \mathfrak{p}, \varphi} \widehat{\mathfrak{u}}(s), \widehat{\vartheta}(s)\right)(\sigma)-g\left(s, \mathcal{D}_{[\sigma]}^{\mathfrak{y}, \mathfrak{p}, \varphi} \mathfrak{u}(s), \vartheta(s)\right)(\sigma)\right| .
\end{aligned}
$$

Thus by $\left(\mathrm{H}_{1}\right)$ we have

$$
\|\widehat{\mathfrak{u}}-\mathfrak{u}\|_{\mathcal{P C}(J)} \leq K \epsilon_{1}+\left[\frac{\varrho_{f}\|\widehat{\mathfrak{u}}-\mathfrak{u}\|_{\mathcal{P C}(J)}+\varrho_{f}^{\prime} \varrho_{g}^{\prime}\|\widehat{\vartheta}-\vartheta\|_{\mathcal{P C}(J)}}{\Gamma(\mathfrak{y}+1)\left(1-\varrho_{f}^{\prime} \varrho_{g}\right)}\right](\varphi(T)-\varphi(0))^{\mathfrak{y}}
$$

By the same technique we get

$$
\|\widehat{\vartheta}-\vartheta\|_{\mathcal{P C}(J)} \leq K \epsilon_{2}+\left[\frac{\varrho_{f} \varrho_{g}\|\widehat{\mathfrak{u}}-\mathfrak{u}\|_{\mathcal{P C}(J)}+\varrho_{g}^{\prime}\|\widehat{\vartheta}-\vartheta\|_{\mathcal{P C}(J)}}{\Gamma(\mathfrak{y}+1)\left(1-\varrho_{f}^{\prime} \varrho_{g}\right)}\right](\varphi(T)-\varphi(0))^{\mathfrak{y}}
$$

It follows that

$$
\|\widehat{\mathfrak{u}}-\mathfrak{u}\|_{\mathcal{P C}(J)}\left(1-\Lambda_{1 f}\right)-\|\widehat{\vartheta}-\vartheta\|_{\mathcal{P C}(J)} \Lambda_{1 g} \leq K \epsilon_{1}
$$

and

$$
\|\widehat{\vartheta}-\vartheta\|_{\mathcal{P C}(J)}\left(1-\Lambda_{2 g}\right)-\|\widehat{\mathfrak{u}}-\mathfrak{u}\|_{\mathcal{P C}(J)} \Lambda_{2 f} \leq K \epsilon_{2}
$$


Inequalities (4.8) and (4.9a) can be rewritten in the matrix form

$$
\left(\begin{array}{cc}
\left(1-\Lambda_{1 f}\right) & -\Lambda_{1 g} \\
-\Lambda_{2 f} & \left(1-\Lambda_{2 g}\right)
\end{array}\right)\left(\begin{array}{c}
\|\widehat{\mathfrak{u}}-\mathfrak{u}\|_{\mathcal{P C}(J)} \\
\|\widehat{\vartheta}-\vartheta\|_{\mathcal{P C}()}
\end{array}\right) \leq\left(\begin{array}{c}
\epsilon_{1} K \\
\epsilon_{2} K
\end{array}\right) .
$$

By simple computations this inequality becomes

$$
\left(\begin{array}{c}
\|\widehat{\mathfrak{u}}-\mathfrak{u}\|_{\mathcal{P C}(J)} \\
\|\widehat{\vartheta}-\vartheta\|_{\mathcal{P C}()}
\end{array}\right) \leq \frac{1}{\Delta}\left(\begin{array}{cc}
\left(1-\Lambda_{2 g}\right) & \Lambda_{1 g} \\
\Lambda_{2 f} & \left(1-\Lambda_{1 f}\right)
\end{array}\right) \times\left(\begin{array}{c}
\epsilon_{1} K \\
\epsilon_{2} K
\end{array}\right) .
$$

This leads to

$$
\begin{aligned}
\|\widehat{\mathfrak{u}}-\mathfrak{u}\|_{\mathcal{P C}(J)} & \leq \frac{\left(1-\Lambda_{2 g}\right) \epsilon_{1} K+\Lambda_{1 g} \epsilon_{2} K}{\Delta}, \\
\|\widehat{\vartheta}-\vartheta\|_{\mathcal{P C}()} & \leq \frac{\Lambda_{2 f} \epsilon_{1} K+\left(1-\Lambda_{1 f}\right) \epsilon_{2} K}{\Delta} .
\end{aligned}
$$

Thus

$$
\begin{aligned}
\|(\widehat{\mathfrak{u}}, \widehat{\vartheta})-(\mathfrak{u}, \vartheta)\|_{\mathcal{B}} & \leq\|\widehat{\mathfrak{u}}-\mathfrak{u}\|_{\mathcal{P C}()}+\|\widehat{\vartheta}-\vartheta\|_{\mathcal{P C}()} \\
& \leq \frac{2-\Lambda_{2 g}+\Lambda_{1 g}+\Lambda_{2 f}-\Lambda_{1 f}}{\Delta} \epsilon K \\
& \leq \mathcal{M} \epsilon,
\end{aligned}
$$

where $\epsilon=\max \left\{\epsilon_{1}, \epsilon_{2}\right\}$ and $\mathcal{M}=\frac{2-\Lambda_{2 g}+\Lambda_{1 g}+\Lambda_{2 f}-\Lambda_{1 f}}{\Delta} K$. Hence by inequality (4.10) and Definition 4.1 the solution of system (1.1) is Ulam-Hyers stable. Next, by setting $\lambda_{\phi}=\epsilon \mathcal{M}$ such that $\lambda_{\phi}(0)=0$ system (1.1) is generalized Ulam-Hyers stable.

\section{An example}

Consider the following problem:

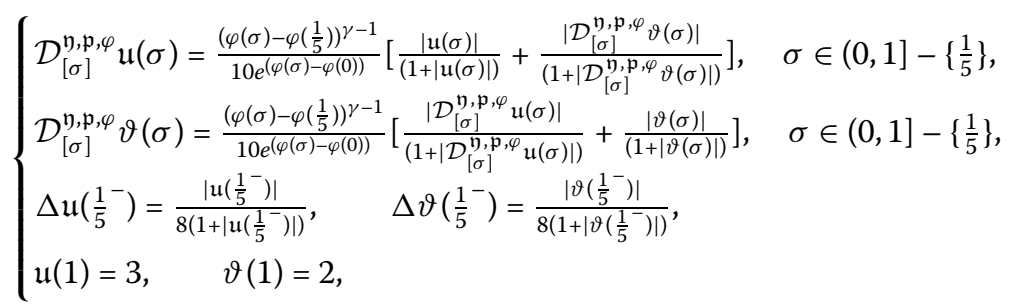

Here $\mathfrak{y}=\frac{1}{3}, \mathfrak{p}=\frac{1}{2}, \gamma=\frac{2}{3}, w_{1}=3, w_{2}=2$. Set $\varphi(\sigma)=e^{\sigma}$.

Example 5.1 Define $f, g:(0,1] \times \mathbb{R}^{2} \rightarrow \mathbb{R}$ as

$$
\begin{aligned}
& f\left(\sigma, \mathfrak{u}(\sigma), \mathcal{D}_{0}^{\mathfrak{y}, \mathfrak{p}, \varphi} \vartheta(\sigma)\right)=\frac{\left(\varphi(\sigma)-\varphi\left(\frac{1}{5}\right)\right)^{\gamma-1}}{10 e^{(\varphi(\sigma)-\varphi(0))}}\left[\frac{|\mathfrak{u}(\sigma)|}{(1+|\mathfrak{u}(\sigma)|)}+\frac{\left|\mathcal{D}_{[\sigma]}^{\mathfrak{y}, \mathfrak{p}, \varphi} \vartheta(\sigma)\right|}{\left(1+\left|\mathcal{D}_{[\sigma]}^{\mathfrak{y}, \mathfrak{p}, \varphi} \vartheta(\sigma)\right|\right)}\right] \\
& g\left(\sigma, \mathcal{D}_{0}^{\mathfrak{y}, \mathfrak{p}, \varphi} \mathfrak{u}(\sigma), \vartheta(\sigma)\right)=\frac{\left(\varphi(\sigma)-\varphi\left(\frac{1}{5}\right)\right)^{\gamma-1}}{10 e^{(\varphi(\sigma)-\varphi(0))}}\left[\frac{\left|\mathcal{D}_{[\sigma]}^{\mathfrak{y}, \mathfrak{p}, \varphi} \mathfrak{u}(\sigma)\right|}{\left(1+\left|\mathcal{D}_{[\sigma]}^{\mathfrak{y}, \mathfrak{p}, \varphi} \mathfrak{u}(\sigma)\right|\right)}+\frac{|\vartheta(\sigma)|}{(1+|\vartheta(\sigma)|)}\right]
\end{aligned}
$$


and $Z_{1}, Z_{2}: \mathbb{R} \rightarrow \mathbb{R}$ by

$$
Z_{1}(\mathfrak{u})=\frac{|\mathfrak{u}|}{8(1+|\mathfrak{u}|)}
$$

and

$$
Z_{2}(\vartheta)=\frac{|\vartheta|}{8(1+|\vartheta|)}
$$

Then, for $(\mathfrak{u}, \vartheta),(\widehat{\mathfrak{u}}, \widehat{\vartheta}) \in \mathbb{R} \times \mathbb{R}$, we have

$$
\begin{aligned}
& |f(\sigma, \mathfrak{u}(\sigma), \vartheta(\sigma))-f(\sigma, \widehat{\mathfrak{u}}(\sigma), \widehat{\vartheta}(\sigma))| \leq \frac{1}{10}|\mathfrak{u}(\sigma)-\widehat{\mathfrak{u}}(\sigma)|+\frac{1}{10}|\vartheta(\sigma)-\widehat{\vartheta}(\sigma)|, \\
& |g(\sigma, \mathfrak{u}(\sigma), \vartheta(\sigma))-g(\sigma, \widehat{\mathfrak{u}}(\sigma), \widehat{\vartheta}(\sigma))| \leq \frac{1}{10}|\mathfrak{u}(\sigma)-\widehat{\mathfrak{u}}(\sigma)|+\frac{1}{10}|\vartheta(\sigma)-\widehat{\vartheta}(\sigma)|,
\end{aligned}
$$

and

$$
\begin{aligned}
& \left|Z_{1}(\mathfrak{u})-Z_{1}(\widehat{\mathfrak{u}})\right| \leq \frac{1}{8}|\mathfrak{u}(\sigma)-\widehat{\mathfrak{u}}(\sigma)|, \\
& \left|Z_{2}(\vartheta)-Z_{2}(\widehat{\vartheta})\right| \leq \frac{1}{8}|\vartheta(\sigma)-\widehat{\vartheta}(\sigma)| .
\end{aligned}
$$

Here $\varrho_{f}=\varrho_{f}^{\prime}=\varrho_{g}=\varrho_{g}^{\prime}=\omega_{f}=\omega_{f}^{\prime}=\omega_{g}=\omega_{g}^{\prime}=\frac{1}{10}$ and $L_{Z_{1}}=L_{Z_{2}}=\frac{1}{8}$. From the given data we deduce that conditions $\left(H_{1}\right),\left(H_{2}\right)$, and $\left(H_{3}\right)$ hold. Thus all the conditions of Theorem 3.1 are satisfied. Therefore problem (1.1) has at least one solution on [0,1]. Moreover, we have $\rho_{1}=\rho_{2}=0,1$ and $\mathcal{Q}=0.48<1$ Thus all conditions of Theorem 3.2 are satisfied. Therefore problem (1.1) has a unique solution on $[0,1]$.

Finally, for $\epsilon=\max \left\{\epsilon_{1}, \epsilon_{2}\right\}>0$, we find that the inequalities

$$
\begin{aligned}
& \left|\mathcal{D}_{[\sigma]}^{\mathfrak{y}, \mathfrak{p}, \varphi} \widehat{\mathfrak{u}}(\sigma)-f\left(\sigma, \widehat{\mathfrak{u}}(\sigma), \mathcal{D}_{[\sigma]}^{\mathfrak{y}, \mathfrak{p}, \varphi} \widehat{\vartheta}(\sigma)\right)\right| \leq \epsilon_{1}, \\
& \left|\mathcal{D}_{[\sigma]}^{\mathfrak{y}, \mathfrak{p}, \varphi} \widehat{\vartheta}(\sigma)-f\left(\sigma, \mathcal{D}_{0}^{\mathfrak{y}, \mathfrak{p}, \varphi} \widehat{\mathfrak{u}}(\sigma), \widehat{\vartheta}(\sigma)\right)\right| \leq \epsilon_{2}
\end{aligned}
$$

are satisfied. Then equation (4.5) is Ulam-Hyers stable with

$$
\|(\widehat{\mathfrak{u}}, \widehat{\vartheta})-(\mathfrak{u}, \vartheta)\|_{\mathcal{B}} \leq \mathcal{M} \epsilon, \quad \varkappa \in J .
$$

where

$$
\mathcal{M}=2.3>0 .
$$

\section{Concluding remarks}

We obtained the existence, uniqueness, and UH stability of solutions for a new problem of $\varphi$-Hilfer FDEs with impulse conditions. Our investigations were based on the reduction of FDEs to FIEs and application the standard Leray-Schauder and Banach fixed point theorems. The acquired results in this paper are more general and cover many of the parallel problems that contain particular cases of the function $\varphi$, because our proposed system contains a global fractional derivative that integrates many classic fractional derivatives; for instance, for various values of a function $\varphi$ and parameter $\mathfrak{p}$, the coupled system 


\title{
(1.1) includes coupled systems of FDEs involving the Hilfer, Hadamard, Katugampola, and many other fractional derivative operators, which are described in the introduction.
}

\begin{abstract}
Acknowledgements
The authors would like to thank the referees for their careful reading of the manuscript and insightful comments. We would also like to acknowledge the valuable comments and suggestions from the editors, which vastly contributed to improvement of the presentation of the paper.
\end{abstract}

Funding

Not applicable.

Availability of data and materials

The authors declare that all data and materials in this paper are available and veritable.

Ethics approval and consent to participate

Not applicable.

Competing interests

The authors declare that they have no competing interests.

Consent for publication

Not applicable.

\section{Authors' contributions}

MAA: Writing original draft, conceptualization, writing review and editing, methodology. SKP: Supervision, review, and editing. All authors read and approved the final manuscript.

\section{Publisher's Note}

Springer Nature remains neutral with regard to jurisdictional claims in published maps and institutional affiliations.

Received: 17 January 2021 Accepted: 21 June 2021 Published online: 29 July 2021

\section{References}

1. Abdeljawad, T.: Fractional operators with generalized Mittag-Leffler kernels and their iterated differintegrals. Chaos 29, 023102 (2019) https://doi.org/10.1063/1.5085726

2. Abdeljawad, T.: Fractional difference operators with discrete generalized Mittag-Leffler kernels. Chaos Solitons Fractals 126, 315-324 (2019)

3. Abdeljawad, T., Baleanu, D.: On fractional derivatives with generalized Mittag-Leffler kernels. Adv. Differ. Equ. 2018 $468(2018)$

4. Abdo, M.S., Abdeljawad, T., Ali, S.M., Shah, K., Jarad, F.: Existence of positive solutions for weighted fractional order differential equations. Chaos Solitons Fractals 141, 110341 (2020)

5. Abdo, M.S., Abdeljawad, T., Shah, K., Jarad, F.: Study of impulsive problems under Mittag-Leffler power law. Heliyon 6(10), e05109 (2020)

6. Abdo, M.S., Shah, K., Panchal, S.K., Wahash, H.A.: Existence and Ulam stability results of a coupled system for terminal value problems involving $\psi$-Hilfer fractional operator. Adv. Differ. Equ. 2020(1), 316 (2020)

7. Abdo, M.S., Thabet, S.T.M., Ahmad, B.: The existence and Ulam-Hyers stability results for $\psi$-Hilfer fractional integrodifferential equations. J. Pseudo-Differ. Oper. Appl. 11, 1757-1780 (2020). https://doi.org/10.1007/s11868-020-00355-x

8. Agarwal, R.P., Benchohra, M., Hamani, S.: A survey on existence results for boundary value problems of nonlinear fractional differential equations and inclusions. Acta Appl. Math. 109(3), 973-1033 (2010)

9. Ahmad, B., Alghanmi, M., Nieto, J.J., Alsaedi, A.: On impulsive nonlocal integro-initial value problems involving multi-order Caputo-type generalized fractional derivatives and generalized fractional integrals. Adv. Differ. Equ. 2019(1), 247 (2019)

10. Ahmad, M., Zada, A., Wang, X.: Existence, Uniqueness and Stability of Implicit Switched Coupled Fractional Differential Equations of $\psi$-Hilfer Type. Int. J. Nonlinear Sci. Numer. Simul. 1(ahead-of-print) (2020)

11. Al-Mayyahi, S.Y., Abdo, M.S., Redhwan, S.S., Abood, B.N.: Boundary value problems for a coupled system of Hadamard-type fractional differential equations. IAENG Int. J. Appl. Math. 51(1), 1-10 (2021)

12. Ali, A., Shah, K., Jarad, F.: Ulam-Hyers stability analysis to a class of nonlinear implicit impulsive fractional differential equations with three point boundary conditions. Adv. Differ. Equ. 2019(1), 7 (2019)

13. Ali, A., Shah, K., Jarad, F., et al.: Existence and stability analysis to a coupled system of implicit type impulsive boundary value problems of fractional-order differential equations. Adv. Differ. Equ. 2019, 101 (2019). https://doi.org/10.1186/s13662-019-2047-y

14. Aljaaidi, T.A., Pachpatte, D.B.: The Minkowski's inequalities via $\psi$-Riemann-Liouville fractional integral operators. Rend. Circ. Mat. Palermo (2) Suppl. (2020). https://doi.org/10.1007/s12215-020-00539-w

15. Almalahi, M.A., Abdo, M.S., Panchal, S.K.: On the theory of fractional terminal value problem with $\psi$-Hilfer fractional derivative. AIMS Math. 5(5), 4889 (2020). https://doi.org/10.3934/math.2020312

16. Almalahi, M.A., Abdo, M.S., Panchal, S.K.: Existence and Ulam-Hyers stability results of a coupled system of $\psi$-Hilfer sequential fractional differential equations. Results Appl. Math. 10, 100142 (2021).

https://doi.org/10.1016/j.rinam.2021.100142 
17. Almalahi, M.A., Abdo, M.S., Panchal, S.K.: Existence and Ulam-Hyers-Mittag-Leffler stability results of $\psi$-Hilfer nonlocal Cauchy problem. Rend. Circ. Mat. Palermo (2) Suppl. 70, 57-77 (2021). https://doi.org/10.1007/s12215-020-00484-8

18. Almalahi, M.A., Panchal, S.K.: On the theory of $\psi$-Hilfer nonlocal Cauchy problem. J. Sib. Fed. Univ. Math. Phys. 14(2), 159-175 (2021). https://doi.org/10.17516/1997-1397-2021-14-2-161-177

19. Almalahi, M.A., Panchal, S.K., Jarad, F.: Stability results of positive solutions for a system of $\psi$-Hilfer fractional differential equations. Chaos Solitons Fractals 147, 110931 (2021). https://doi.org/10.1016/.j.chaos.2021.110931

20. Almalahi, M.A., Panchal, S.K., Jarad, F., Abdeljawad, T.: Ulam-Hyers-Mittag-Leffler stability for tripled system of weighted fractional operator with TIME delay. Adv. Differ. Equ. 2021, 299 (2021). https://doi.org/10.1186/s13662-021-03455-0

21. Almeida, R:: A Caputo fractional derivative of a function with respect to another function. Commun. Nonlinear Sci. Numer. Simul. 44, 460-481 (2017)

22. Almeida, R., Malinowska, A.B., Odzijewicz, T.: Fractional differential equations with dependence on the Caputo-Katugampola derivative. J. Comput. Nonlinear Dyn. 11(6) (2016). https://doi.org/10.1115/1.4034432

23. Alsulami, H.H., Ntouyas, S.K., Agarwal, R.P., Ahmad, B., Alsaedi, A.: A study of fractional-order coupled systems with a new concept of coupled non-separated boundary conditions. Bound. Value Probl. 2017, 68 (2017)

24. Atangana, A.: Fractal-fractional differentiation and integration: connecting fractal calculus and fractional calculus to predict complex system. Chaos Solitons Fractals 102, 396-406 (2017)

25. Atangana, A., Baleanu, D.: New fractional derivative with non-local and non-singular kernel. Therm. Sci. 20(2), 757-763 (2016)

26. Atangana, A., Baleanu, D.: New fractional derivatives with non-local and non-singular kernel: theory and application to heat transfer model. Therm. Sci. 20(2), 763-769 (2016)

27. Benchohra, M., Seba, D.: Impulsive fractional differential equations in Banach spaces. Electron. J. Qual. Theory Differ. Equ. 8(1), 14 (2009)

28. Benchohra, M., Slimani, B.A.: Existence and uniqueness of solutions to impulsive fractional differential equations. Electron. J. Differ. Equ. 2009, 10 (2009)

29. Deimling, K.: Nonlinear Functional Analysis. Springer, New York (1985)

30. Derbazi, C., Baitiche, Z., Abdo, M.S., Abdeljawad, T.: Qualitative analysis of fractional relaxation equation and coupled system with $\psi$-Caputo fractional derivative in Banach spaces. AIMS Math. 6(3), 2486-2509 (2021)

31. Granas, A., Dugundji, J.: Fixed Point Theory. Springer, Berlin (2013)

32. Guo, T.L.: Nonlinear impulsive fractional differential equations in Banach spaces. Topol. Methods Nonlinear Anal. 42(1), 221-232 (2013)

33. Jarad, F., Abdeljawad, T.: Generalized fractional derivatives and Laplace transform. Discrete Contin. Dyn. Syst., Ser. S 2019, 709 (2019)

34. Jarad, F., Abdeljawad, T., Baleanu, D.: Caputo-type modification of the Hadamard fractional derivatives. Adv. Differ. Equ. 2012(1), 142 (2012)

35. Kharade, J.P., Kishor, D.K.: On the impulsive implicit $\psi$-Hilfer fractional differential equations with delay. Math. Methods Appl. Sci. 43(4), 1938-1952 (2020)

36. Kilbas, A.A., Srivastava, H.M.. Trujillo, J.J.: Theory and Applications of Fractional Differential Equations. Elsevier, Amsterdam (2006)

37. Oliveira, E., Sousa, J.V.C.: Ulam-Hyers-Rassias stability for a class of fractional integro-differential equations. Results Math. 73(3), 111 (2018)

38. Podlubny, I.: Fractional Differential Equations. Academic Press, New York (1999)

39. Rus, I.A.: Ulam stabilities of ordinary differential equations in a Banach space. Carpath. J. Math. 26, 103-107 (2010)

40. Samko, S.G., Kilbas, A.A., Marichev, O.I. Fractional Integrals and Derivatives. Gordon \& Breach, Yverdon (1993)

41. Sousa, J.V.C., de Oliveira, C.E.: On the $\psi$-Hilfer fractional derivative. Commun. Nonlinear Sci. Numer. Simul. 60, 72-91 (2018)

42. Sousa, J.V.C., de Oliveira, C.E.: On the Ulam-Hyers-Rassias stability for nonlinear fractional differential equations using the $\psi$-Hilfer operator. J. Fixed Point Theory Appl. 20(3), 96 (2018)

43. Sousa, J.V.C., de Oliveira, C.E.: A Gronwall inequality and the Cauchy-type problem by means of $\psi$-Hilfer operator. Differ. Equ. Appl. 11(1), 87-106 (2019)

44. Wang, J., Feckan, M., Zhou, Y.: A survey on impulsive fractional differential equations. Fract. Calc. Appl. Anal. 19(4), 806-831 (2016)

\section{Submit your manuscript to a SpringerOpen ${ }^{\mathcal{O}}$ journal and benefit from:}

- Convenient online submission

- Rigorous peer review

- Open access: articles freely available online

- High visibility within the field

- Retaining the copyright to your article

Submit your next manuscript at $\boldsymbol{~ s p r i n g e r o p e n . c o m ~}$ 